\title{
Identification of Time-Varying Pilot Control Behavior in Multi-Axis Control Tasks
}

\author{
Peter M.T. Zaal* \\ San Jose State University Research Foundation, San Jose, CA \\ and \\ Barbara T. Sweet ${ }^{\dagger}$ \\ NASA Ames Research Center, Moffett Field, CA
}

\begin{abstract}
Recent developments in fly-by-wire control architectures for rotorcraft have introduced new interest in the identification of time-varying pilot control behavior in multi-axis control tasks. In this paper a maximum likelihood estimation method is used to estimate the parameters of a pilot model with time-dependent sigmoid functions to characterize timevarying human control behavior. An experiment was performed by 9 general aviation pilots who had to perform a simultaneous roll and pitch control task with time-varying aircraft dynamics. In 8 different conditions, the axis containing the time-varying dynamics and the growth factor of the dynamics were varied, allowing for an analysis of the performance of the estimation method when estimating time-dependent parameter functions. In addition, a detailed analysis of pilots' adaptation to the time-varying aircraft dynamics in both the roll and pitch axes could be performed. Pilot control behavior in both axes was significantly affected by the time-varying aircraft dynamics in roll and pitch, and by the growth factor. The main effect was found in the axis that contained the time-varying dynamics. However, pilot control behavior also changed over time in the axis not containing the time-varying aircraft dynamics. This indicates that some cross coupling exists in the perception and control processes between the roll and pitch axes.
\end{abstract}

\section{Nomenclature}

$\begin{array}{llll}A_{d} & \text { sinusoid amplitude, deg } & L & \text { likelihood function, - } \\ B & \text { bias } & M & \text { parameter function time of maximum growth, s } \\ C_{\Theta \Theta} & \text { Cramér-Rao lower bound } & M_{\Theta \Theta} & \text { Fisher information matrix } \\ e & \text { tracking error signal, deg } & m & \text { number of samples } \\ f & \text { probability density function, }- & N & \text { number of points } \\ f_{d} & \text { disturbance forcing function, deg } & n & \text { pilot remnant signal, deg } \\ G & \text { parameter function growth rate, } \mathrm{s}^{-1} & n_{d} & \text { forcing function frequency integer factor } \\ H_{c} & \text { aircraft dynamics response } & P_{1} & \text { initial parameter function value, }- \\ H_{o l} & \text { open-loop response } & P_{2} & \text { final parameter function value, }- \\ H_{p} & \text { pilot response } & s & \text { Laplace variable } \\ H_{n} & \text { remnant filter } & T_{c} & \text { vehicle dynamics time constant, s } \\ j & \text { imaginary unit } & T_{l} & \text { pilot lead time constant, s } \\ K_{c} & \text { vehicle dynamics gain, } & T_{m} & \text { measurement time, s } \\ K_{n} & \text { pilot remnant gain, }- & t & \text { time, s } \\ K_{v} & \text { pilot visual gain, }- & u & \text { pilot control signal, deg } \\ k & \text { sinusoid index } & & \end{array}$

*Aerospace Engineer, Human Systems Integration Division, Moffett Field, CA, 94035; peter.m.t.zaal@nasa.gov. Member AIAA.

${ }^{\dagger}$ Aerospace Engineer, Human Systems Integration Division, Moffett Field, CA, 94035; barbara.t.sweet@nasa.gov. Member AIAA. 


$\begin{array}{ll}\text { Symbols } & \\ \alpha & \text { line search parameter, - } \\ \epsilon & \text { prediction error, deg } \\ \phi & \text { roll angle, deg } \\ \phi_{d} & \text { sinusoid phase shift, rad } \\ \varphi_{m} & \text { phase margin, deg } \\ \Theta & \text { parameter vector } \\ \theta & \text { pitch angle, deg }\end{array}$

$\begin{array}{ll}\sigma^{2} & \text { variance } \\ \tau_{e} & \text { effective time delay, s } \\ \tau_{v} & \text { pilot visual delay, } \mathrm{s} \\ \omega & \text { frequency, rad s} \\ \omega_{c} & \text { crossover frequency, rad s } \\ \omega_{n m} & \text { pilot neuromuscular frequency, } \mathrm{rad} \mathrm{s}^{-1} \\ \omega_{d} & \text { sinusoid frequency, rad s } \\ \zeta_{n m} & \text { pilot neuromuscular damping, }-\end{array}$

\section{Introduction}

In many aircraft control tasks, pilots need to adapt their control strategy to controlled aircraft dynamics that change over time. Examples of control tasks with time-varying dynamics are: the manual control of a tilt-rotor aircraft during the transition from hovering to forward flight, and the manual control of rotorcraft with variable speed rotors or aircraft with reconfigurable flight control systems. ${ }^{1}$ Currently, not much is known about the adaptation of pilot skill based control behavior to time-varying dynamics, as most previous research on control behavior focused on control tasks with constant dynamics. In addition, most previous research focused on pilot control behavior in single-axis control tasks, while most pilot control tasks require simultaneous control inputs in multiple axes.

Recent developments in fly-by-wire control architectures for rotorcraft have introduced new interest in the identification of time-varying pilot control behavior in multi-axis control tasks. ${ }^{2}$ Most research in the past on the time-varying aspect of human control behavior has been devoted to the development of complex mathematical models to describe adaptations of behavior over time. ${ }^{3}$ An accurate estimate of the parameters of these models would give valuable insight by quantifying how a human adapts to time-varying controlled dynamics. However, because of the complexity of the models it is not possible to accurately estimate their parameters from measured data. In addition, there are insufficient techniques for the identification of timevarying human control behavior and the estimation of time-varying pilot model parameters.

Techniques for the identification and prediction of human control behavior in control tasks with constant dynamics are well established. ${ }^{4-7}$ In recent years, work on the identification of time-varying pilot control behavior focused on the use of wavelet transforms to identify time-varying pilot frequency response functions and derived parameters such as crossover frequencies and phase margins. ${ }^{8-10}$ However, when applied to human control data, the wavelet transform is known to produce inaccurate results, as it is very sensitive to the relatively high pilot remnant levels. ${ }^{11}$ Furthermore, the use of wavelets does not allow for the estimation of time-varying pilot model parameters directly. A second step is required, fitting the pilot model frequency response to the wavelet frequency response. ${ }^{6}$

In a recent study, maximum likelihood estimation (MLE) has been used to estimate time-varying pilot model parameters directly from time-domain data. ${ }^{11}$ The time dependency of the pilot model parameters was introduced by estimating constant parameters on a sliding time window. This approach produced relatively noisy results and required significant computational power as the calculations have to be performed at every time step.

In this paper an MLE method is used to estimate the parameters of a pilot model with time-dependent sigmoid functions to characterize time-varying human control behavior from experiment data in a single step. This method promises to be far more efficient to the time-windowing MLE method previously used and possibly allows for more accurate results. An experiment was conducted using 9 general aviation pilots who performed a simultaneous roll and pitch control task with time-varying aircraft dynamics. In 8 different conditions the axis containing the time-varying dynamics and growth factor of the dynamics were varied, allowing for an analysis of the performance of the MLE method when estimating time-dependent parameter functions. In addition, a detailed analysis of pilots' adaptation in different axes of control to the time-varying aircraft dynamics was performed.

The multi-axis control task and the time-varying aircraft and pilot model dynamics will be discussed in Sec. II, followed by the adopted MLE method used for the estimation of time-varying pilot model parameters in Sec. III. Next, the experiment setup will be discussed in Sec. IV. The results will be given in Sec. V and discussed in Sec. VI. Finally, conclusions will be given in Sec. VII. 


\section{Multi-Axis Control Task}

\section{A. Control Loop}

Fig. 1 depicts the multi-axis disturbance-rejection task that was used to analyze time-varying pilot control behavior in this study. A pilot controls the roll and pitch attitude $(\phi$ and $\theta)$ of an aircraft with dynamics $H_{c}$ by making control inputs $\left(u_{\phi}\right.$ and $\left.u_{\theta}\right)$ with a joystick. The aircraft dynamics do not have any cross coupling between the roll and pitch axes. The pilot's task is to keep the aircraft level by keeping the roll and pitch angles $(\phi$ and $\theta)$ as small as possible at all times, while they are perturbed by disturbances $\left(f_{d \phi}\right.$ and $\left.f_{d \theta}\right)$. The disturbed roll and pitch angels $\left(\phi_{d}\right.$ and $\left.\theta_{d}\right)$ are displayed on the primary flight display depicted in Fig. 2.

In single-loop control tasks, pilot skill-based control behavior can be modeled by a linear response function $H_{p}$ and a remnant signal $n$ that accounts for non-linear behavior. As the aircraft dynamics in roll and pitch do not have any cross coupling, it is assumed that pilot control behavior in this task can be modeled by two independent linear response functions $\left(H_{p \phi}\right.$ and $\left.H_{p \theta}\right)$ and two independent remnant signals $\left(n_{\phi}\right.$ and $\left.n_{\theta}\right)$. The resulting control diagram structure of the pilot-vehicle system consists of two independent single-loop control loops (Fig. 1). Hence, the system can be termed multiple single-loop. The assumption of two separate control-loops in a control task with uncoupled dynamics has been adopted in previous studies and was found to be appropriate for data analysis purposes. ${ }^{12}$

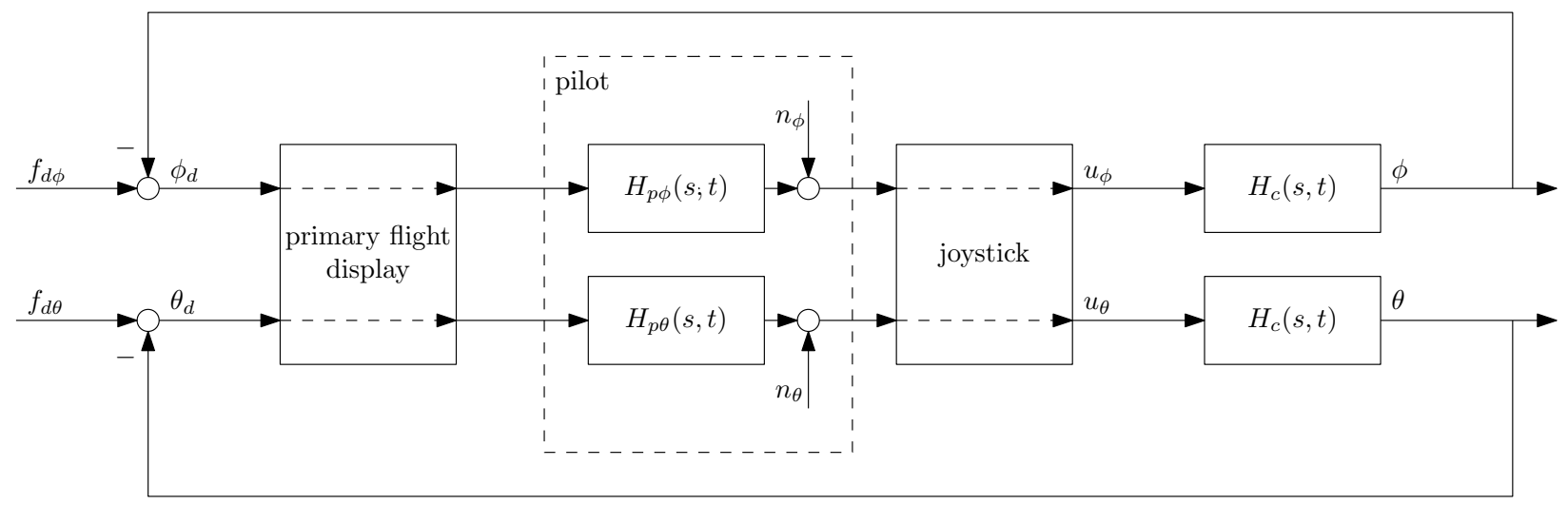

Figure 1. Multi-axis closed-loop control task.

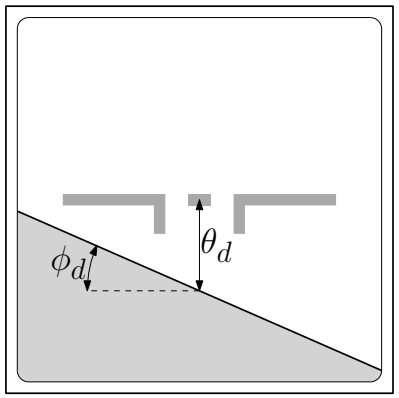

Figure 2. Primary flight display.

A typical example of the multi-axis control task depicted in Fig. 1 is longitudinal and lateral stabilization of an aircraft in straight, wings-level, horizontal flight using aileron and elevator control inputs. Techniques for the identification and parameterization of pilot response functions in this type of control task are well established for vehicle dynamics $H_{c}$ that are constant over time. ${ }^{4,13,14}$ However, the vehicle dynamics in Fig. 1 change over time. Such time-varying dynamics can, for example, be introduced by an aircraft control system that adapts to different flight conditions or phases of flight. As a result, pilots will adapt their control behavior over time to maintain adequate levels of performance and stability margins. The goal of this study is to quantify this adaptation in pilot control behavior by estimating time-varying pilot model parameters using maximum likelihood estimation. ${ }^{11}$ 


\section{B. Time-Dependent Parameter Functions}

Many types of parameter functions can be used to describe a time dependency in the pilot model parameters. However, the function needs to be able to describe the expected transient response of a certain parameter. The most appropriate parameter function needs to be determined with prior knowledge of the change in controlled dynamics and by carefully inspecting the measured data. In practice, the complexity of the function will depend on many variables, such as the overshoot and settling time of the transient response. Care should be taken that not too many additional parameters are introduced by the parameter function, as this can lead to over parameterization of the complete pilot model, resulting in less accurate parameter estimates. Furthermore, it will be more difficult to find a global optimum parameter set, as multiple parameter combinations may result in similar model responses.

The parameter function considered in this study is the generalized logistic function, a widely-used sigmoid function. Sigmoid functions are characterized by their S-shape, describing the transition from an initial value to a final value. These functions are often used for learning curve and growth modeling. The generalized logistic function as a function of time $t$ is defined by:

$$
P(t)=P_{1}+\frac{P_{2}-P_{1}}{1+e^{-G(t-M)}}
$$

where $P_{1}$ is the initial pilot model parameter value and $P_{2}$ the final parameter value. The time of maximum growth is defined by $M$ and the growth rate by $G$. Fig. 3 depicts the generalized logistic function for variations of the time-of-maximum-growth and growth-rate parameters. For $G \rightarrow \infty$ the parameter function converges to a step function, allowing for the modeling of an instant change in pilot control behavior. Note that for a value of $G=10$ the function already approaches a step function (Fig. 3b). This means that this parameter might be difficult to estimate, depending on its real value and the sampling frequency of the data, as it has no upper limit (e.g. a growth rate of 100 or 1000 are indistinguishable).

(a) $M$ variation $\left(P_{1}=0.1, P_{2}=0.4, G=0.5\right)$

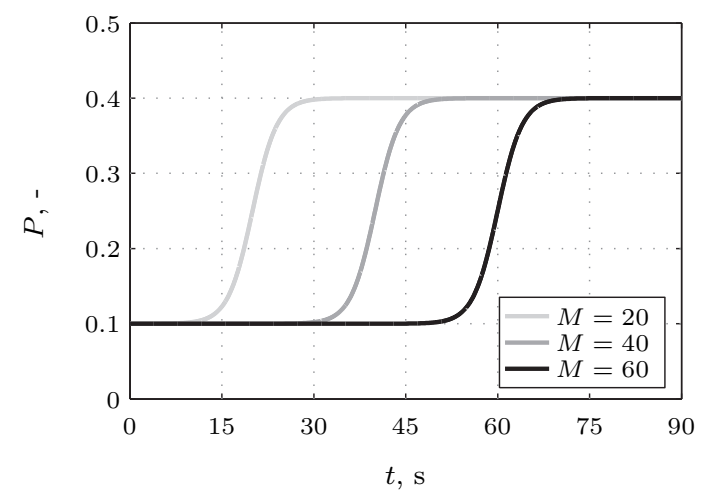

(b) $G$ variation $\left(P_{1}=0.1, P_{2}=0.4, M=50\right)$

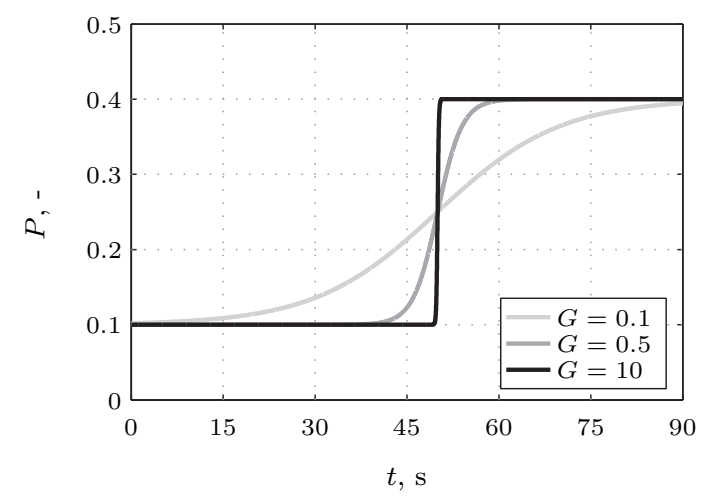

Figure 3. Generalized logistic function parameter variation.

\section{Time-varying Dynamics}

The time-varying aircraft roll and pitch dynamics in Fig. 1 are defined by:

$$
H_{c}(s, t)=\frac{K_{c}(t)}{s^{2}+T_{c}(t) s}
$$

where the gain $K_{c}(t)=K_{c 1}+\left[K_{c 2}-K_{c 1}\right] /\left[1+e^{-G(t-M)}\right]$ and the time constant $T_{c}(t)=T_{c 1}+\left[T_{c 2}-T_{c 1}\right] /[1+$ $\left.e^{-G(t-M)}\right]$ are time-varying parameters defined by the parameter function given in Eq. (1). The initial and final parameter function values are $K_{c 1}=90.0, K_{c 2}=30.0, T_{c 1}=6.0 \mathrm{~s}$, and $T_{c 2}=0.2 \mathrm{~s}$. The time of maximum growth is $M=50.0 \mathrm{~s}$ and the growth rate is either $G=0.5 \mathrm{~s}^{-1}$ or $G=100.0 \mathrm{~s}^{-1}$, as will be explained in Sec. IV. The time of maximum growth and the growth rate are equivalent for both time-varying parameters of the aircraft dynamics. Given the parameter functions described above, the aircraft dynamics will change over time from $H_{c 1}=90.0 /\left(s^{2}+6.0 s\right)$ to $H_{c 2}=30.0 /\left(s^{2}+0.2 s\right)$. 
(a) Aircraft dynamics magnitude

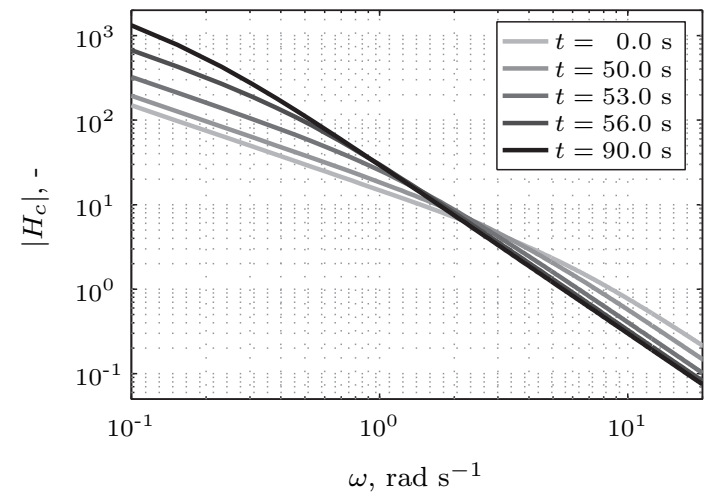

(b) Aircraft dynamics phase

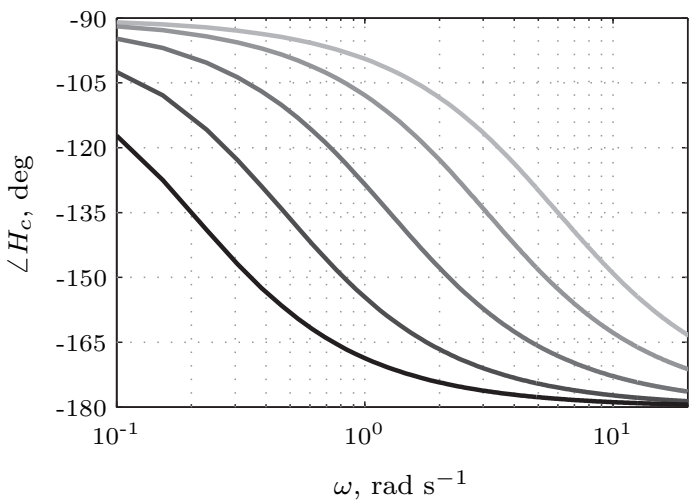

Figure 4. Frequency response of the aircraft dynamics at different time instances.

Fig. 4 depicts the time-varying aircraft dynamics for different time instances. Note that the dynamics will change from mostly stable dynamics $(1 / s)$ to mostly unstable dynamics $\left(1 / s^{2}\right)$ in the human crossover frequency range $(1-5 \mathrm{rad} / \mathrm{s})$. The crossover model theorem states that human operators adapt their control behavior such that the pilot-vehicle open-loop response in the crossover region can be described by:

$$
H_{o} l(s)=H_{p}(s) H_{c}(s) \approx \frac{\omega_{c}}{s} e^{-s \tau_{e}}
$$

with $\omega_{c}$ the crossover frequency and $\tau_{e}$ a time delay lumping the human processing lags. ${ }^{4}$ In order to comply with Eq. (3) and given the variations in $H_{c}$, the equalization dynamics of the pilot model need to contain a lead time constant. The pilot lead time constant is expected to increase over time (lead is generated at increasingly lower frequencies) as the time constant of the aircraft dynamics decreases. Based on previous research, the pilot gain is also expected to change over time. ${ }^{15}$ The time-varying pilot model capable of modeling this adaptation in pilot control behavior is defined by:

$$
H_{p}(s, t)=\overbrace{K_{v}(t)\left[1+T_{l}(t) s\right]}^{\text {equalization }} \overbrace{e^{-s \tau_{v}} \frac{\omega_{n m}^{2}}{\omega_{n m}^{2}+2 \zeta_{n m} \omega_{n m} s+s^{2}}}^{\text {limitations }}
$$

The equalization dynamics of the pilot model consist of a time-varying gain $K_{v}(t)=K_{v 1}+\left[K_{v 2}-\right.$ $\left.K_{v 1}\right] /\left[1+e^{-G(t-M)}\right]$ and a time-varying lead time constant $T_{l}(t)=T_{l 1}+\left[T_{l 2}-T_{l 1}\right] /\left[1+e^{-G(t-M)}\right]$. The pilot limitations, defined by the time delay $\tau_{v}$, neuromuscular damping $\zeta_{n m}$, and neuromuscular frequency $\omega_{n m}$ - are assumed to be constant. Previous research found that the limitation parameters are approximately equivalent for a pilot controlling dynamics similar to $H_{c 1}$ or similar to $H_{c 2} \cdot{ }^{15}$ The time of maximum growth and the growth rate of both parameter functions are assumed to be equal. This means a parameter vector

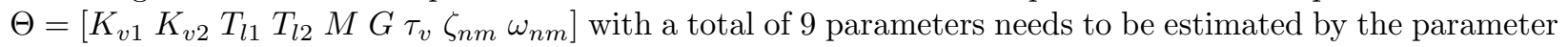
estimation procedure.

\section{Disturbance Forcing Functions}

Both disturbance forcing functions are a sum of sines constructed using the following equation:

$$
f_{d \phi, d \theta}(t)=\sum_{k=1}^{N_{d}} A_{d}(k) \sin \left[\omega_{d}(k) t+\phi_{d \phi, d \theta}(k)\right]
$$

with $N_{d}=10$ the number of sine waves, and $\omega_{d}, A_{d}$ and $\phi_{d \phi, d \theta}$ the frequency, amplitude and phase shift of the $k^{\text {th }}$ sine wave, respectively. The length of an experiment run is $T=90.00 \mathrm{~s}$. The measurement time used to construct the forcing functions is $T_{m}=81.92 \mathrm{~s}$. With a data sampling frequency of $100 \mathrm{~Hz}$, this measurement time contains the highest power-of-two measurements $\left(N_{m}=8192\right)$ in the total length of an experiment run. The sinusoid frequencies $\omega_{d}(k)$ were all integer multiples of the measurement-time base 
Table 1. Disturbance forcing function properties.

\begin{tabular}{rrclrc}
\hline \hline$k,-$ & $n_{d},-$ & $\omega_{d}, \mathrm{rad} \mathrm{s}^{-1}$ & $A_{d}, \mathrm{deg}$ & $\phi_{d \phi}, \mathrm{rad}$ & \multicolumn{1}{c}{$\phi_{d \theta}, \mathrm{rad}$} \\
\hline 1 & 3 & 0.230 & 1.186 & 0.974 & -0.753 \\
2 & 5 & 0.384 & 1.121 & -3.026 & 1.564 \\
3 & 8 & 0.614 & 0.991 & 0.744 & 0.588 \\
4 & 13 & 0.997 & 0.756 & -2.300 & -0.546 \\
5 & 22 & 1.687 & 0.447 & 2.984 & 0.674 \\
6 & 34 & 2.608 & 0.245 & -2.513 & -1.724 \\
7 & 53 & 4.065 & 0.123 & 2.211 & -1.963 \\
8 & 86 & 6.596 & 0.061 & 1.004 & -2.189 \\
9 & 139 & 10.661 & 0.036 & -2.255 & 0.875 \\
10 & 229 & 17.564 & 0.025 & 2.210 & 0.604 \\
\hline \hline
\end{tabular}

frequency, $\omega_{m}=2 \pi / T_{m}=0.0767 \mathrm{rad} / \mathrm{s}$, and were covering the frequency range of human control (0.1-18 $\mathrm{rad} / \mathrm{s}$ ). The spacing between the frequencies was approximately equal on a logarithmic scale.

The absolute value of a second-order low-pass filter at a sinusoid frequency was used to determine the amplitudes of the individual sine waves:

$$
A_{d}(k)=\left|\frac{\left[1+0.1 j \omega_{d}(k)\right]^{2}}{\left[1+0.8 j \omega_{d}(k)\right]^{2}}\right|
$$

The second-order low-pass filter ensures reduced amplitudes at higher frequencies, yielding a control task that is not overly difficult. The final amplitude distributions were scaled to produce roll and pitch disturbance forcing functions with a standard deviation of $1.5 \mathrm{deg}$.

To determine the forcing function phase distributions, a large number of random phase sets were generated. The set yielding a signal with a probability distribution closest to a Gaussian distribution, without leading to excessive peaks, was selected. ${ }^{16}$ The characteristics of the forcing functions are summarized in Table 1. Note that the the roll and pitch disturbance forcing functions contain the same frequencies and amplitudes. However, the phase distributions of the two forcing functions were different.

\section{Identification of Time-Varying Pilot Control Behavior}

\section{A. Maximum Likelihood Estimation}

The MLE procedure adopted in this research is similar to the one described in Ref. 7. Some modifications are applied to facilitate the time-dependent parameter functions of the pilot model.

The MLE procedure calculates an estimate $\hat{\Theta}$ of the pilot model parameter vector $\Theta$ by maximizing a likelihood function. The likelihood function $L(\Theta)$ is defined as the joint conditional probability density function of the prediction error for $m$ measurements of the pilot control signal $u(k)$ :

$$
L(\Theta)=f[\epsilon(1), \epsilon(2), \ldots, \epsilon(k), \ldots, \epsilon(m) \mid \Theta]
$$

The prediction error, indicated as $\epsilon(k)$ in Eq. (7), is defined as the difference between the measured pilot control signal $u(k)$ and the control signal from the pilot model $\hat{u}(k)$ at discrete instants. When the remnant is assumed to be an additive zero-mean Gaussian white noise signal, the conditional probability density function for one measurement of $\epsilon(k)$ is given by:

$$
f(\epsilon(k) \mid \Theta)=\frac{1}{\sqrt{2 \pi \sigma_{n}^{2}}} e^{-\frac{\epsilon^{2}(k)}{2 \sigma_{n}^{2}}}
$$

The set of parameters that maximizes the likelihood function is the maximum likelihood estimate of the parameter vector $\Theta$. To create a more straightforward optimization problem, it is common practice to minimize the negative natural logarithm of the likelihood function instead of maximizing $L(\Theta)$. The parameter vector resulting in the global minimum of the negative log-likelihood function is then the maximum likelihood estimate $\hat{\Theta}_{M L}$. For the single-output pilot model defined in Eq. (4), combining Eq. (7) and Eq. (8) results into the following expression for the maximum likelihood parameter estimate: 


$$
\hat{\Theta}_{M L}=\underset{\Theta}{\arg \min }-\ln L(\Theta)=\underset{\Theta}{\arg \min }\left[\frac{m}{2} \ln \sigma_{n}^{2}+\frac{1}{2 \sigma_{n}^{2}} \sum_{k=1}^{m} \epsilon^{2}(k)\right]
$$

The minimum of this function is found using an unconstrained gradient-based Gauss-Newton optimization. The iterative parameter update equation for the Gauss-Newton optimization is given by:

$$
\hat{\Theta}(i+1)=\hat{\Theta}(i)-\alpha(i) M_{\Theta \Theta}^{-1}[\hat{\Theta}(i)] \frac{\partial L[\hat{\Theta}(i)]}{\partial \Theta}
$$

where $\alpha$ is the line-search parameter determined at each iteration before the actual parameter update. The optimal $\alpha$ is determined by calculating the likelihood for different values of $\alpha$ between 0 and 1 . The $\alpha$ that results in the biggest decrease of the negative natural logarithm of the likelihood function is used for the parameter update. This ensures the most rapid minimization of the likelihood function.

The gradients of the likelihood function with respect to all parameters, $\partial L / \partial \Theta$, are calculated using a simple three-point finite difference approximation:

$$
\frac{\partial L[\hat{\Theta}(i)]}{\partial \Theta}=\frac{L[\hat{\Theta}(i)+\Delta \Theta]-L[\hat{\Theta}(i)-\Delta \Theta]}{2 \Delta \Theta}
$$

where $\Delta \Theta=0.01$ is a small increment to the parameters in the parameter vector. The Fisher information matrix, indicated with the symbol $M_{\Theta \Theta}$ in Eq. (10), is given by:

$$
M_{\Theta \Theta}=\frac{1}{\sigma_{n}^{2}} \sum_{k=1}^{m}\left[\frac{\partial \epsilon(k)}{\partial \Theta}\right]^{2}
$$

The Fisher information matrix is symmetrical and needs to be positive definite (i.e., full rank), as it needs to be inverted for the parameter update equation. The inverse of the Fisher information matrix yields the Cramér-Rao lower bound (CRLB), which determines the lowest achievable variance of the parameter estimates:

$$
C_{\Theta \Theta}=M_{\Theta \Theta}^{-1}
$$

Both the Fisher information matrix and the first-order gradient of the likelihood function vary with the magnitude of the estimation error $\epsilon$, yielding parameter update steps of larger magnitude if estimation errors are larger.

As described in Ref. 7, a genetic algorithm is used to find a solution of the parameter vector close to the global minimum. This solution is then used as an initial parameter vector in the unconstrained gradientbased Gauss-Newton optimization. This significantly increases the chance of finding the global optimum solution of the parameter estimation problem.

\section{B. Bias and Variance}

A Monte Carlo analysis with 140 simulations was performed to determine the bias and variance characteristics of the proposed MLE method in combination with the control task and aircraft dynamics discussed in Sec. II. The simulations were performed using a single control loop of the diagram in Fig. 1. Note that both the roll and pitch control loops in the diagram are equivalent. Fig. 5 depicts the simplified control loop used in the simulations.

The time-varying aircraft dynamics $H_{c}$ and pilot dynamics $H_{p}$ used in the simulations are described in Sec. II.C. The growth rate of the time-varying aircraft dynamics was set to 0.5. The parameters of the time-varying pilot model were selected using data from a test experiment: $K_{v 1}=0.09, K_{v 2}=0.07, T_{l 1}=0.4$ $\mathrm{s}, T_{l 2}=1,2 \mathrm{~s}, M=50.0 \mathrm{~s}, G=0.5 \mathrm{~s}^{-1}, \tau_{v}=0.28 \mathrm{~s}, \zeta_{n m}=0.35$, and $\omega_{n m}=11.25 \mathrm{rad} / \mathrm{s}$.

The pilot remnant $n_{\theta}$ was simulated using a low-pass filtered white noise signal $w$. The remnant filter was defined by: $H_{n}=K_{n} /(0.2 s+1)$. Three different levels of pilot remnant were used to investigate the effect of remnant intensity on the bias and variance of the parameter estimates. The remnant intensity is defined by: $P_{n}=\sigma^{2}(n) / \sigma^{2}(u)$. The filter gain $K_{n}$ was selected to induce remnant intensities of $P_{n}=0.05$, $P_{n}=0.15$, and $P_{n}=0.25$.

Figs. $6 \mathrm{a}$ and $6 \mathrm{~b}$ depict the relative bias and variance, respectively, of the pilot model parameters for different levels of remnant intensity. The relative bias and variance are calculated by dividing the bias and 


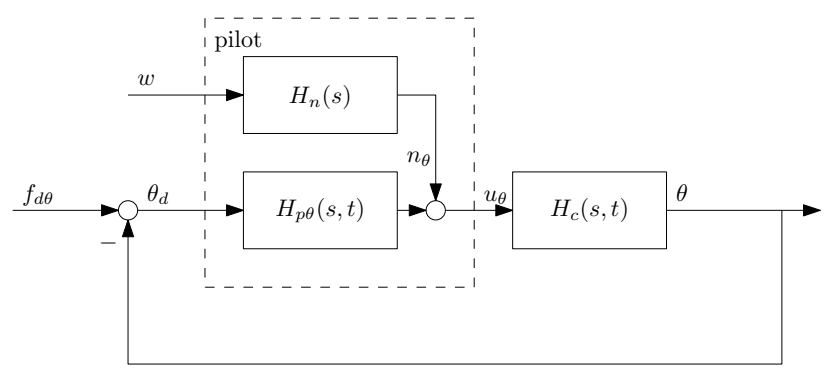

Figure 5. Single-loop control task used for simulations.

variance of each parameter by the value of the parameter itself. From Fig. 6a it can be observed that the bias increases for most of the parameters as the remnant intensity increases. Furthermore, the bias of $K_{v 2}$, $T_{l 2}$, and $G$ is higher compared to the other variables.

Fig. $6 \mathrm{~b}$ indicates that the relative variance of the parameters also increases as the remnant intensity increases, an expected result. The figure further indicates that the variance of the final parameter function values $K_{v 2}$ and $T_{l 2}$ is higher compared to the initial values $K_{v 1}$ and $T_{l 1}$. Finally, it can be observed that the growth rate $G$ has the highest variance of all parameters.

Table 2 provides the CRLB variance of the pilot model parameters averaged over 140 simulations for different values of remnant intensity. Although the values in this table provide the lowest achievable variance, they still provide a good indication of the relative estimation accuracy of every pilot model parameter.

Table 2. CRLB variance of the time-varying pilot model parameters (140 simulations).

\begin{tabular}{|c|c|c|c|c|c|c|c|c|c|}
\hline \multirow[b]{2}{*}{$P_{n}$} & \multicolumn{9}{|c|}{$C_{\Theta \Theta}$} \\
\hline & $K_{v 1}$ & $K_{v 2}$ & $T_{l 1}$ & $T_{l 2}$ & $M$ & $G$ & $\tau_{v}$ & $\zeta_{n m}$ & $\omega_{n m}$ \\
\hline 0.05 & $5.49 \times 10^{-6}$ & $1.56 \times 10^{-5}$ & $3.78 \times 10^{-4}$ & $4.67 \times 10^{-3}$ & $3.48 \times 10^{-1}$ & $3.62 \times 10^{-2}$ & $1.71 \times 10^{-5}$ & $2.79 \times 10^{-4}$ & $7.02 \times 10^{-2}$ \\
\hline 0.15 & $4.81 \times 10^{-6}$ & $1.27 \times 10^{-5}$ & $3.49 \times 10^{-4}$ & $4.99 \times 10^{-3}$ & $3.67 \times 10^{-1}$ & $3.90 \times 10^{+6}$ & $1.56 \times 10^{-5}$ & $2.57 \times 10^{-4}$ & $6.87 \times 10^{-2}$ \\
\hline 0.25 & $3.77 \times 10^{-6}$ & $1.10 \times 10^{-5}$ & $2.85 \times 10^{-4}$ & $8.52 \times 10^{-3}$ & $5.96 \times 10^{-1}$ & $4.86 \times 10^{+7}$ & $1.31 \times 10^{-5}$ & $2.07 \times 10^{-4}$ & $6.08 \times 10^{-2}$ \\
\hline
\end{tabular}

The table indicates that the final parameter function values have higher lower bounds compared to the initial parameter function values, implicating that these variables possibly have a lower accuracy. This was also apparent from Fig. 6b. Next, the growth rate appears to have the highest lower bound values of all model parameters. The low expected accuracy of this parameter was also apparent from Fig. 6b.

Summarizing, the results of the Monte Carlo analysis indicate that the final parameter function variables $K_{v 2}$ and $T_{l 2}$, and the growth rate $G$ will most likely have the lowest accuracy when estimated from experiment data. However, the results presented are for this particular set of pilot model parameters. The true pilot model parameter bias and variance characteristics will depend on many factors, such as pilot performance, remnant characteristics, and control behavior.

(a) Pilot model parameter bias

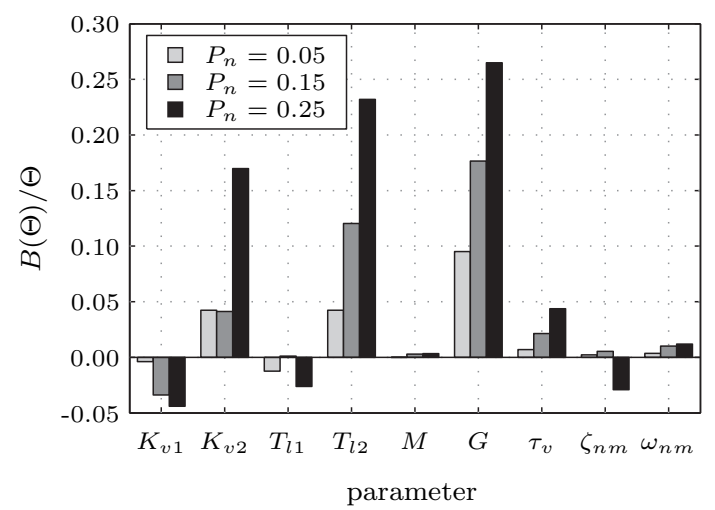

(b) Pilot model parameter variance

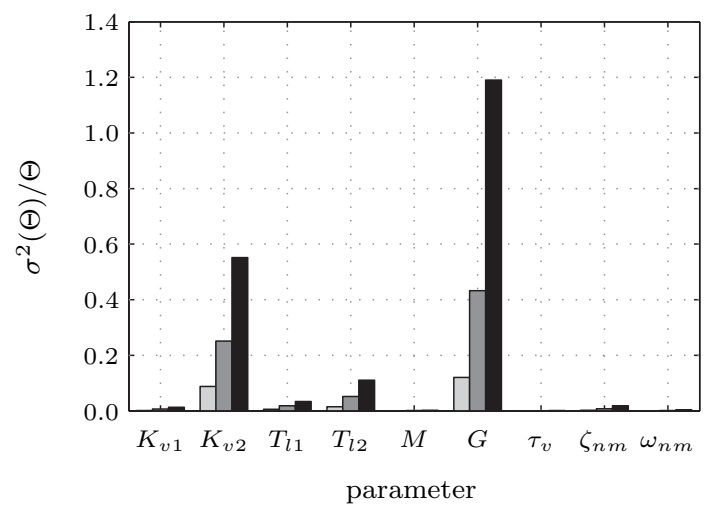

Figure 6. Relative bias and variance of the time-varying pilot model parameters (140 simulations). 


\section{Experiment Setup}

The experiment was performed with two goals in mind; to investigate how pilot control behavior is affected by time-varying aircraft dynamics in a multi-axis control task and to evaluate the performance of the proposed maximum likelihood estimation method when estimating the parameters of a time-varying pilot model with time-dependent parameter functions in different controlled axes and for different growth rates of the controlled aircraft dynamics.

\section{A. Method}

\section{Independent Variables}

Three independent variables with two levels each were varied in the experiment: 1$)$ roll-axis dynamics $\left(H_{c 1}\right.$ or $\left.\left.H_{c 1} \rightarrow H_{c 2}\right), 2\right)$ pitch-axis dynamics $\left(H_{c 1}\right.$ or $\left.H_{c 1} \rightarrow H_{c 2}\right)$, and 3) growth rate of the change in dynamics $(G=0.5$ or $G=100.0)$. A full-factorial design was implemented, resulting in 8 conditions in total. However, two of these conditions are equivalent, as the growth rate does not have an effect when the dynamics in both the roll and pitch axes remain constant $\left(H_{c 1}\right)$. This means only 7 distinctive conditions result from this full-factorial design. As a reference, an additional condition was performed with constant dynamics $H_{c 2}$ in both the roll and pitch axes. The aircraft dynamics $H_{c 1}$ and $H_{c 2}$ are defined in Sec. II.C.

The 8 experiment conditions are listed in Table 3. Conditions 1 and 2 -referred to by the symbols $\mathrm{C} 1$ and $\mathrm{C} 2$ - have constant dynamics in both the roll and pitch axes. Conditions 3 to 5 (C3-C5) have time-varying dynamics with a growth rate of 0.5 in one or both of the axes. This growth rate results in a gradual change from $H_{c 1}$ to $H_{c 2}$ in about $20 \mathrm{~s}$. In conditions 6 to 8 (C6-C8), the axis containing the time-varying dynamics are equivalent to $\mathrm{C} 3$ to $\mathrm{C} 5$. However, the time-varying dynamics have a growth rate of 100.0, resulting in an almost instant (step-like) change from $H_{c 1}$ to $H_{c 2}$.

Table 3. Experiment conditions.

\begin{tabular}{|c|c|c|c|c|}
\hline Condition & Roll dynamics & Pitch dynamics & \multicolumn{2}{|c|}{ Growth rate } \\
\hline $\mathrm{C} 1$ & $H_{c 1}$ & $H_{c 1}$ & & \\
\hline $\mathrm{C} 2$ & $H_{c 2}$ & $H_{c 2}$ & & \\
\hline C3 & $H_{c 1} \rightarrow H_{c 2}$ & $H_{c 1}$ & 0.5 & \\
\hline $\mathrm{C} 4$ & $H_{c 1}$ & $H_{c 1} \rightarrow H_{c 2}$ & 0.5 & \\
\hline C5 & $H_{c 1} \rightarrow H_{c 2}$ & $H_{c 1} \rightarrow H_{c 2}$ & 0.5 & \\
\hline C6 & $H_{c 1} \rightarrow H_{c 2}$ & $H_{c 1}$ & 100.0 & \\
\hline $\mathrm{C} 7$ & $H_{c 1}$ & $H_{c 1} \rightarrow H_{c 2}$ & 100.0 & \\
\hline $\mathrm{C} 8$ & $H_{c 1} \rightarrow H_{c 2}$ & $H_{c 1} \rightarrow H_{c 2}$ & 100.0 & 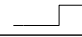 \\
\hline
\end{tabular}

\section{Apparatus}

Fig. 2 shows the primary flight display (PFD) used to provide pilots with attitude information. The PFD was depicted on a ViewSonic G225f CRT monitor. This monitor has a viewing area with a diagonal of 20 inch and was set to a resolution of $1600 \times 1200 \mathrm{px}$. The size of the PFD was $400 \times 400 \mathrm{px}$ and was depicted in the center of the screen. The thickness of the aircraft symbol was $6 \mathrm{px}$ and the thickness of the horizon was $2 \mathrm{px}$. The roll angle was magnified by a factor of 2 on the display. One degree of pitch angle was visible by an 8 px translation of the horizon from the aircraft symbol (see Fig. 2). Pilots were seated approximately $1.8 \mathrm{ft}$ in front of the monitor.

A Logitech Extreme 3D Pro joystick was used to make control inputs. This right-handed joystick has considerable breakout force. Joystick output values in the roll and pitch axes ranged from -1 to 1 , corresponding to full left-right and fore-aft joystick deflections, respectively.

\section{Participants and Instructions}

Nine general aviation pilots participated in the experiment. All were male with an average age of 24.3 years. The average total of flying hours was 773.2 hours with an average of 78.2 flying hours in the last 6 months. All pilots were right handed and only 1 had previous experience with the type of control task used.

Every pilot received a briefing before the start of the experiment. The briefing contained information about the objective of the study. Furthermore, they were given details about the control task, conditions, 
and procedures of the experiment. Pilots were instructed to make control inputs in an active and continuous manner, trying to minimize the roll and pitch angles presented on the visual display to the best of their capabilities. In addition, they were told to focus on minimizing roll and pitch angles at the same time.

\section{Procedures}

All experimental runs had a length of $90 \mathrm{~s}$. The first $8.08 \mathrm{~s}$ of the run were considered the run-in time. During this phase, pilots were able to stabilize the controlled dynamics and adjust to the task. Data from the last $81.92 \mathrm{~s}$ were used as measurement data for the data analysis (see Sec. II.D).

All pilots performed a total of 96 runs. The experiment consisted of 4 segments, each containing 24 runs. Each of the 8 conditions was presented 3 times in every segment. The conditions were presented in a quasi-random order using a Latin-square design. Pilots were not informed about which condition they were performing.

The first two segments were used for pilot training and runs from the last two segments were used for data analysis. After every run, tracking performance - defined as the root mean square (RMS) of the roll and pitch angles - was presented to the pilots to motivate them to constantly perform at their maximum level of performance. In between each segment, the experimenter made sure pilots reached and maintained asymptotic performance; that is, more or less constant RMS values.

Pilots were able to go through the experiment at there own pace. They were able to start the next run by pressing the trigger on the joystick. This allowed them to take breaks when feeling any discomfort. Every pilot was able to complete the experiment within 4 hours.

\section{Dependent Measures}

Several dependent measures were calculated from the measured signals from the experiment. First, pilot performance and control activity in the roll and pitch axes of the disturbance-rejection task were evaluated in terms of the RMS of the attitude and control signals, respectively. Next, the parameters of the timevarying pilot model were estimated using the MLE method discussed in Sec. III. For every participant and condition the experimental data were averaged over six runs before the start of the estimation procedure. The control signal variance accounted for (VAF) was calculated as a measure for the accuracy of the pilot model in describing the measured control signal data. Finally, time-varying pilot-vehicle open-loop responses and corresponding time-varying crossover frequencies and phase margins were calculated.

\section{B. Hypotheses}

Very little research has been performed on the identification of time-varying control behavior in multiaxis control tasks, or the estimation of time-varying parameters using experiment data. However, some hypotheses on the performance of MLE for the estimation of time-varying parameters from experiment data can be formulated based on Sec. III.B. In addition, some hypotheses on pilot adaptation to the time-varying aircraft dynamics can be formulated based on experiments with single-loop control tasks with constant dynamics. ${ }^{15,17}$

In Sec. III.B it was found that the bias and variance of the final parameter function parameters and the growth rate parameter were higher compared to the other parameters of the model. The bias and variance increased along with levels of remnant. It is expected that in a multi-axis task, pilot remnant levels will be higher, as attention needs to be divided between the two axes. This results in a hypothesis that larger variations are expected to be found in $K_{v 2}, T_{l 2}$, and $G$. Furthermore, it is hypothesized that the accuracy of the overall pilot model (determined by the VAF) will be higher for the unstable dynamics $H_{c 2} .{ }^{17}$

In conditions in which the aircraft dynamics change from stable dynamics $H_{c 1}$ to unstable dynamics $H_{c 2}$, it is hypothesized that pilot roll and pitch disturbance-rejection performance will decrease, and roll and pitch control activity will increase over time. ${ }^{15}$ In addition, crossover frequencies will increase and phase margins will decrease. Finally, the pilot visual gain $K_{v}$ and visual lead time constant $T_{l}$ are expected to decrease and increase, respectively, when the dynamics change from $H_{c 1}$ to $H_{c 2} \cdot{ }^{15}$ 
(a) Roll attitude signal

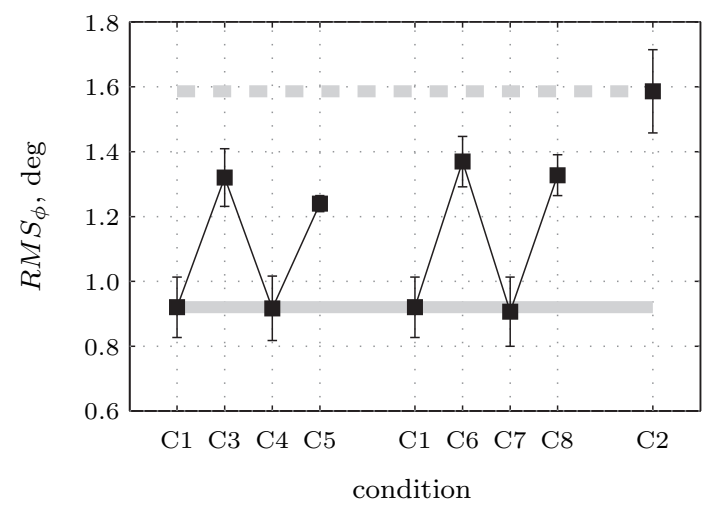

(b) Roll control signal

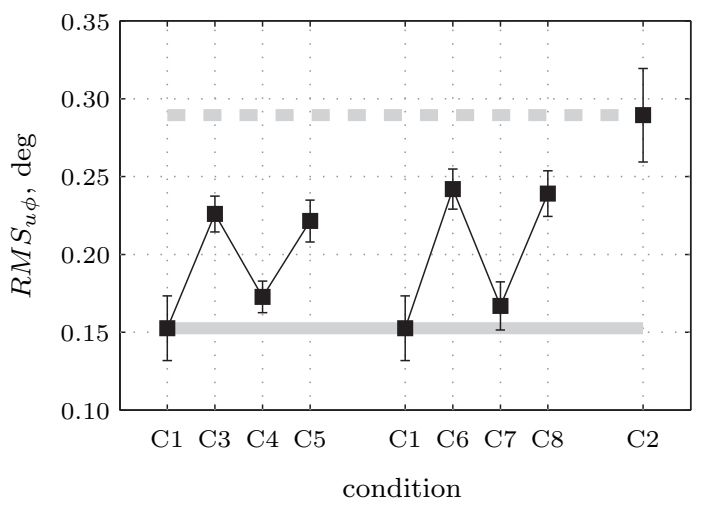

Figure 7. Roll tracking performance and control activity.

\section{Results}

This section provides the combined results of the 9 pilots that participated in the experiment. The error bars in the error bar plots are corrected for between-subject variability by normalizing the subject means across conditions. ${ }^{18}$ The data from the reference conditions $\mathrm{C} 1$ and $\mathrm{C} 2$ are marked by gray continuous and dashed lines, respectively. All the dependent measures of the experiment were analyzed using a repeatedmeasures analysis of variance (ANOVA) to uncover any significant trends.

\section{A. Pilot Performance and Control Activity}

Pilot performance and control activity in the roll axis - defined by the RMS of the roll angle and the RMS of the roll control signal - are depicted in Figs. 7a and 7b. Figs. 8a and 8b provide the same data for the pitch axis. A lower value for the roll or pitch angle RMS indicates a higher performance and a lower value for the control signal RMS indicates a lower control activity.

Fig. 7a indicates that performance is higher for the reference condition $\mathrm{C} 1$ with stable dynamics compared to reference condition $\mathrm{C} 2$ with unstable dynamics, a highly significant effect $[F(1,8)=49.661, p<0.001]$. This was an expected result, also observed in previous studies. ${ }^{15}$ When looking at the effects of the change in roll dynamics on the RMS of the roll angle, a significant deterioration in performance can be observed when there is a change in roll dynamics from $H_{c 1}$ to $H_{c 2}[F(1,8)=41.302, p<0.001]$. The performance in these conditions was approximately half way between the performance for $\mathrm{C} 1$ and $\mathrm{C} 2$. No significant effect was found from a change in pitch dynamics $[F(1,8)=3.252, p>0.05]$. The change in growth factor introduced a significant effect $[F(1,8)=10.757, p<0.05]$. For the higher growth factor of the aircraft dynamics, the performance was slightly worse compared to the lower growth factor. One significant interaction was present between roll dynamics and growth factor $[F(1,8)=15.441, p<0.05]$. For the higher growth factor, the change in roll dynamics introduced a larger decrease in roll performance.

From Fig. 7b the observation can be made that the control activity is significantly higher for condition $\mathrm{C} 2$ compared to $\mathrm{C} 1[F(1,8)=39.329, p<0.001]$. A change in roll dynamics significantly increased roll control activity $[F(1,8)=73.000, p<0.001]$. Although roll control activity also seems slightly increased when only pitch dynamics vary in time $(\mathrm{C} 4$ and $\mathrm{C} 7)$, the overall effect is not significant $[F(1,8)=2.189$, $p>0.05]$. Growth rate did not introduce a significant effect $[F(1,8)=4.419, p>0.05]$. Two interactions between the independent variables were found. The first, between growth rate and roll dynamics $[F(1,8)$ $=16.115, p<0.001]$. This indicates that for the higher growth rate, the control activity increase from the change in roll dynamics is larger. The second interaction occurred between the change in roll and the change in pitch dynamics, reflecting the previously observed increase in control activity when only pitch dynamics vary in time $[F(1,8)=9.564, p<0.05]$.

The results in the pitch axis when the pitch dynamics were changed from stable to unstable dynamics are very similar to the results in the roll axis for a change in roll dynamics. From Fig. 8a it can be observed that disturbance-rejection performance is significantly better in the condition with constant stable dynamics $\mathrm{C} 1$ compared to the condition with constant unstable dynamics $\mathrm{C} 2[F(1,8)=29.778, p<0.01]$. The changing 
(a) Pitch attitude signal

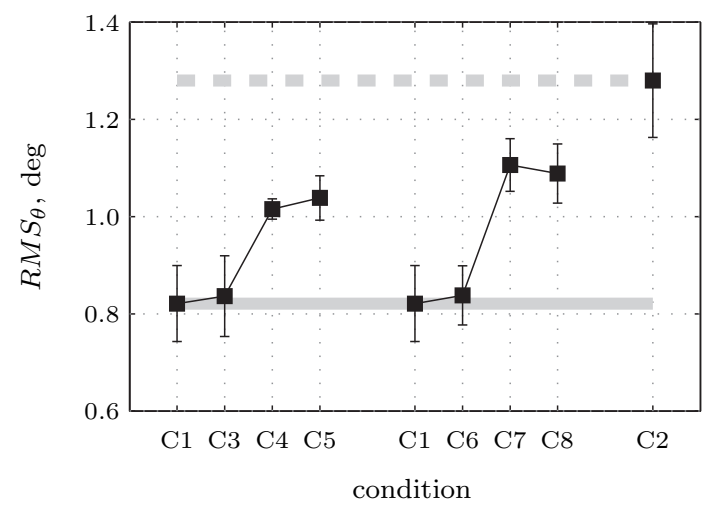

(b) Pitch control signal

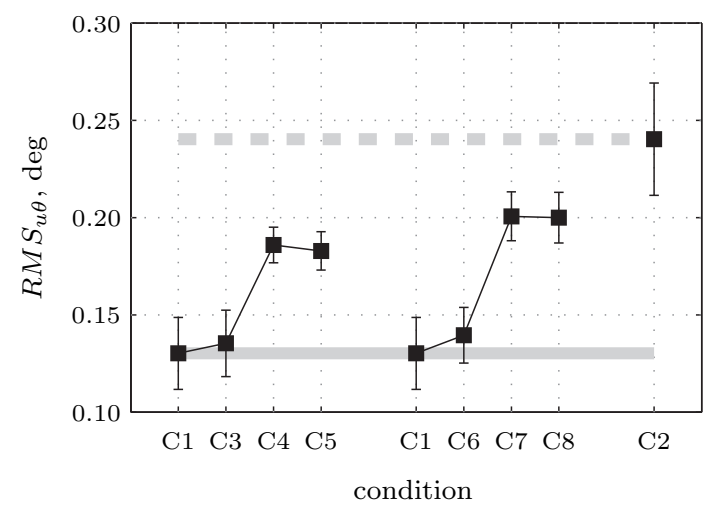

Figure 8. Pitch tracking performance and control activity.

dynamics in pitch $[(\mathrm{C} 4, \mathrm{C} 5)$ and $(\mathrm{C} 7, \mathrm{C} 8)]$ introduced a significant effect in pitch RMS $[F(1,8)=29.004$, $p<0.01]$, but no significant effect was found from the changing roll dynamics $[F(1,8)=1.566, p>0.05]$. The growth rate of the changing aircraft dynamics also affected the pitch disturbance-rejection performance significantly $[F(1,8)=6.406, p<0.05]$. The performance is slightly lower for the dynamics with a higher growth rate. One significant interaction is present in the data between growth rate and pitch dynamics $[F(1$, $8)=17.286, p<0.01]$. The reduction in performance caused by the change in pitch dynamics is slightly larger for the dynamics with a higher growth rate.

Fig. $8 \mathrm{~b}$ indicates that pitch control activity is significantly higher for the condition with constant unstable dynamics compared to the condition with constant stable dynamics $[F(1,8)=29.882, p<0.01]$. The change in roll dynamics did not introduce a significant effect $[F(1,8)=0.463, p>0.05]$. However, the change in pitch dynamics influenced the pitch control activity significantly $[F(1,8)=36.488, p<0.001]$. In the conditions with time-varying dynamics in pitch, the control activity in pitch was significantly higher, approximately halfway between the control activity for $\mathrm{C} 1$ and $\mathrm{C} 2$. In addition, pitch control activity was significantly higher in the conditions with the higher growth rate $[F(1,8)=17.670, p<0.01]$. The larger increase in control activity for the higher growth rate when the pitch dynamics changed over time was revealed by a significant interaction between growth rate and pitch dynamics $[F(1,8)=11.843, p<0.01]$.

Finally, by comparing performance and control activity between the roll and pitch axes (comparing Figs. 7 and 8), it can be observed that the performance in reducing the disturbance was higher and control activity was lower in the pitch axis. These are both significant effects $[F(1,8)=22.218, p<0.01]$ and $[F(1,8)=$ $50.480, p<0.001]$.

\section{B. Pilot Model Variance Accounted For}

The pilot model parameters were estimated using the MLE method discussed in Sec. III. For the conditions with constant dynamics ( $\mathrm{C} 1$ and $\mathrm{C} 2$ ), a pilot model was identified without any time-dependent parameter functions. This simplified the model and reduced the parameter vector to 5 parameters, $\Theta=$ $\left[\begin{array}{lllll}K_{v 1} & T_{l 1} & \tau_{v} & \zeta_{n m} & \omega_{n m}\end{array}\right]$.

Figs. 9 and 10 give the VAF for the roll and pitch control signal, respectively. A higher VAF indicates that a larger percentage of the measured data can be explained by the linear pilot model. In addition to the model with time-dependent parameter functions $\left[H_{p}(s, t)\right]$, the VAF in the conditions with time-varying dynamics was also calculated for the model with constant parameters $\left[H_{p}(s)\right]$. This allows to determine if the pilot model with time-dependent parameter functions is able to describe the data better compared to the pilot model without parameter functions when the aircraft dynamics change over time.

Fig. 9 reveals, as hypothesized in Sec. IV.B, that the VAF in the roll axis is significantly higher for the condition with unstable dynamics $\mathrm{C} 2$ compared to the condition with stable dynamics $\mathrm{C} 1[F(1,8)=25.590$, $p<0.01]$. An investigation of the effects of the change in roll and pitch dynamics and growth rate shows that only a change in roll dynamics introduced a significant effect in the roll VAF. The roll VAF in the conditions with time-varying roll dynamics was significantly higher $[F(1,8)=80.525, p<0.001]$, approximately at the same level of the VAF in condition C2. Comparing the VAF of both pilot models in the conditions with 


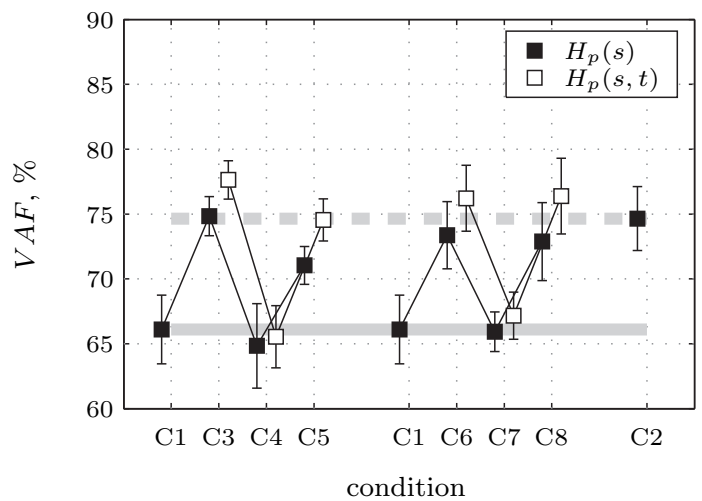

Figure 9. Roll axis variance accounted for.

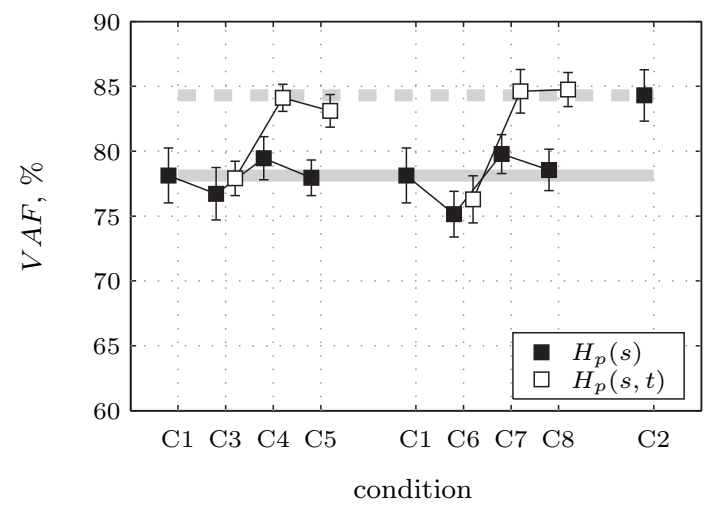

Figure 10. Pitch axis variance accounted for.

time-varying aircraft dynamics (white compared to black squares in conditions C3-C5 and C6-C8), it can be observed that the VAF is slightly higher for the pilot model with time-dependent parameter functions $[F(1$, $8)=51.867, p<0.001]$. This indicates that this model is indeed more suitable to describe the data in the conditions with time-varying aircraft dynamics.

The results in the pitch axis (Fig. 10) are very similar compared to the roll axis. The VAF in C2 is significantly higher compared to $\mathrm{C} 1[F(1,8)=18.329, p<0.01]$. The change in pitch dynamics from stable to unstable dynamics introduced a significant increase in $\operatorname{VAF}[F(1,8)=40.775, p<0.001]$. No significant effects were introduced by the change in roll dynamics or the growth rate. When comparing the two pilot models in the conditions with time-varying aircraft dynamics, it can be observed that the VAF is significantly higher for the model with time-dependent parameter functions $[F(1,8)=91.753, p<0.001]$. Again, this indicates that this model is more suitable to describe the data in the conditions with time-varying aircraft dynamics.

Finally, comparing the VAF between the roll and pitch axes (Fig. 9 compared to Fig. 10), the observation can be made that the VAF in the pitch axis was significantly higher $[F(1,8)=107.690, p<0.001]$. This might indicate that pilots behaved more linear (i.e., introduced less remnant) in the pitch axis, as a higher percentage of the data could be explained by the linear pilot model.

\section{Pilot Model Parameters}

Figs. 11 and 12 present the estimated parameters for the pilot model in roll and pitch, respectively. The parameters estimated in all conditions are depicted by black squares. The parameters that are only available in the conditions with time-varying aircraft dynamics are depicted by white squares. As for these parameters a full-factorial repeated-measures ANOVA cannot be performed, a repeated-measures ANOVA is performed with the time-varying aircraft dynamics (not taking into account roll and pitch) and growth rate as factors.

Results from the ANOVA indicate that, for the roll axis, there is no significant difference in the initial visual gain $K_{v 1}$ between the condition with constant stable dynamics $\mathrm{C} 1$ and the condition with constant unstable dynamics $\mathrm{C} 2[F(1,8)=2.159, p>0.05]$ (see Fig. 11a). No significant effects are found for $K_{v 1}$ for the change in roll or pitch dynamics, or the growth rate of the dynamics. This is an expected result, as $K_{v 1}$ characterizes pilot control behavior for the stable aircraft dynamics $H_{c 1}$ in all conditions, except in C2. For the final visual gain $K_{v 2}$ a significant effect was found for the change in dynamics $[F(2,16)=5.889, p<$ 0.05]. This was also an expected result, because the values for this parameter in conditions with a change in roll dynamics $(\mathrm{C} 3, \mathrm{C} 5, \mathrm{C} 6, \mathrm{C} 8)$ should be closer to the value for $K_{v 1}$ in C2. Comparing $K_{v 1}$ with $K_{v 2}$ for the conditions with time-varying dynamics, a significant lower value for $K_{v 2}$ was found $[F(1,8)=8.604, p<$ $0.05]$.

The visual gain for the pitch pilot model is given in Fig. 12a. The initial visual gain $K_{v 1}$ is significantly lower in $\mathrm{C} 2$ compared to $\mathrm{C} 1[F(1,8)=28.501, p<0.01]$. Again, as expected, no significant effects are found for $K_{v 1}$ for the change in roll or pitch dynamics, or the growth rate. The change in dynamics or the growth rate also did not introduce a significant effect in the final visual gain $K_{v 2}$. The values for $K_{v 2}$ are significantly lower than $K_{v 1}$ for the conditions with a change in dynamics $[F(1,8)=69.017, p<0.001]$. In these conditions the value for $K_{v 2}$ more closely resembled the value for $K_{v 1}$ in $\mathrm{C} 2$. This was expected for the 
(a) Visual gain

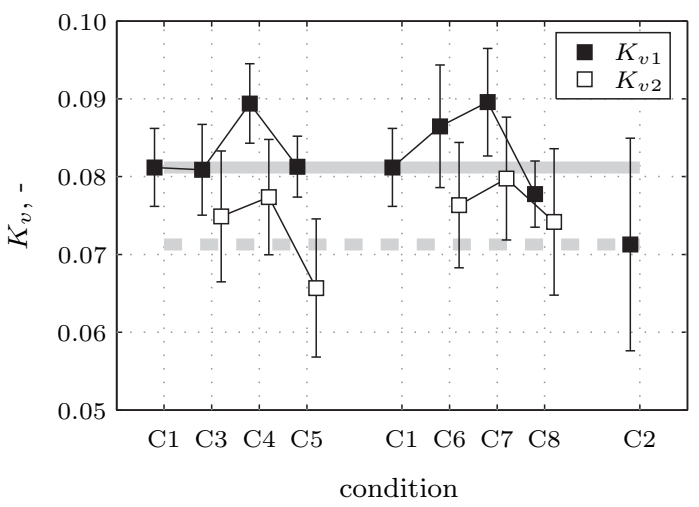

(c) Tim of maximum growth

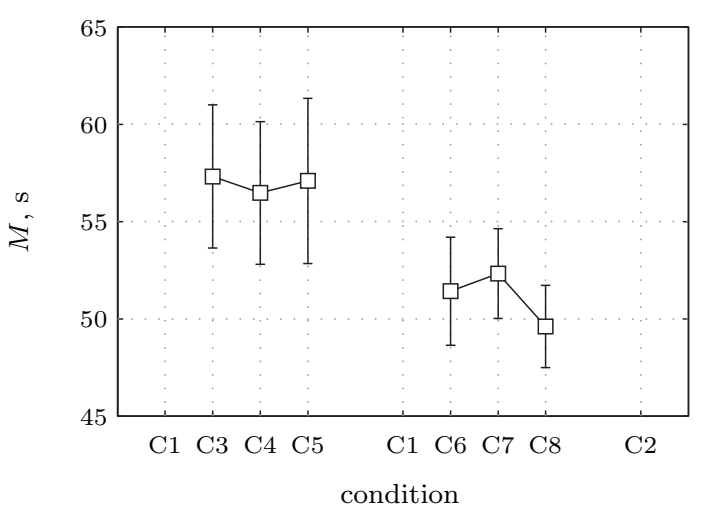

(b) Lead time constant

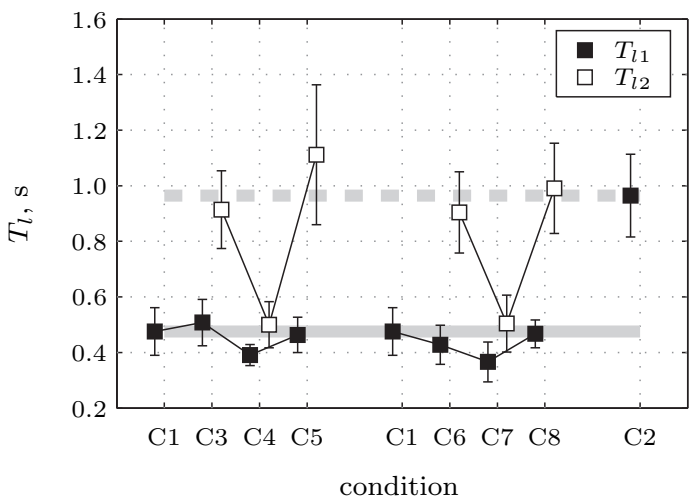

(d) Growth rate

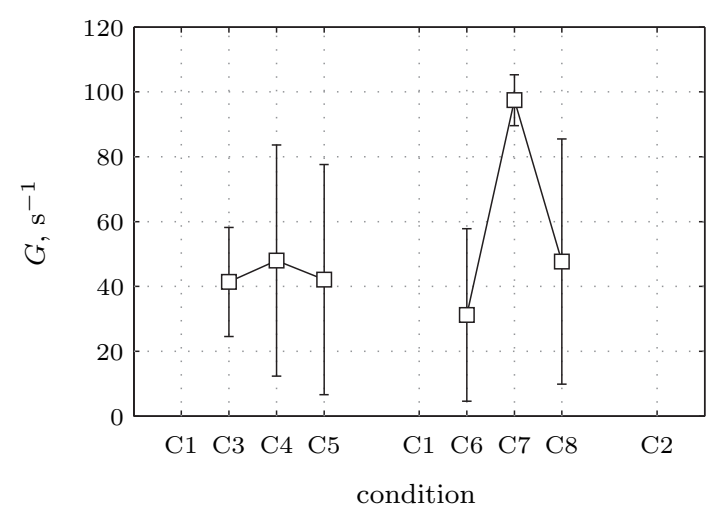

(e) Visual delay

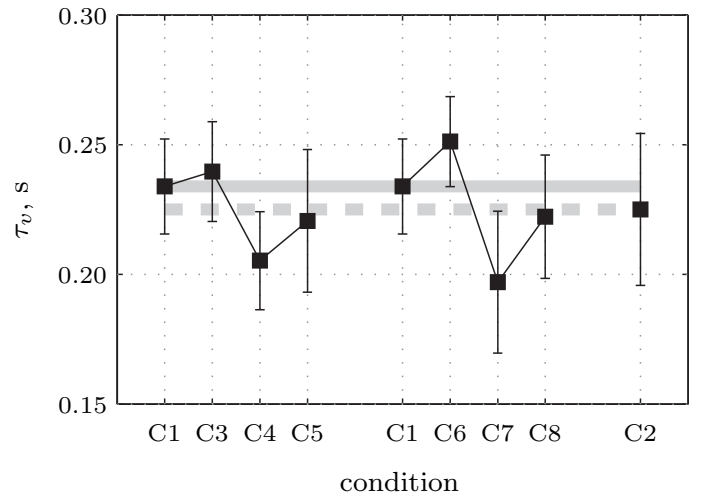

(f) Neuromuscular damping

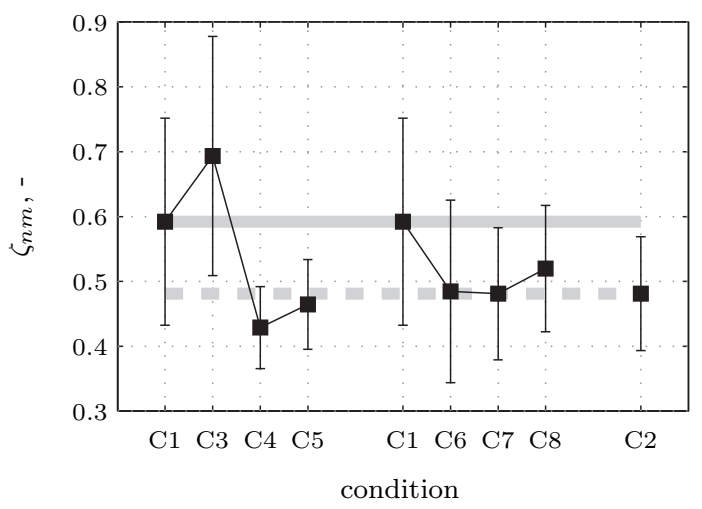

(g) Neuromuscular frequency

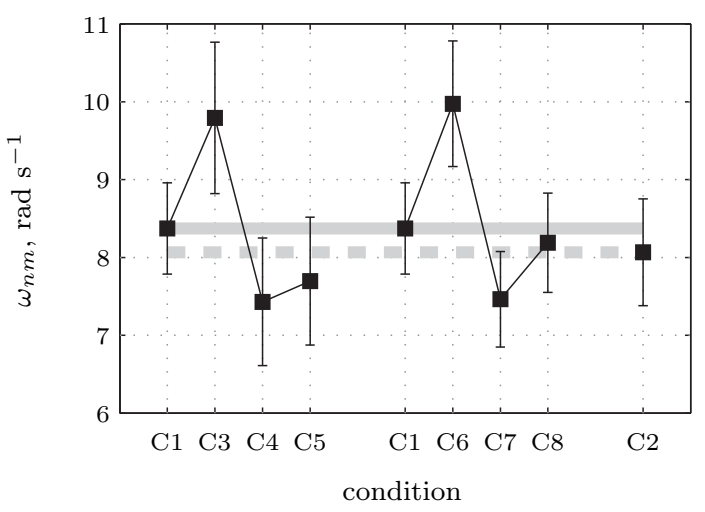

Figure 11. Means and $95 \%$ confidence intervals of the roll pilot model parameters. 
(a) Visual gain

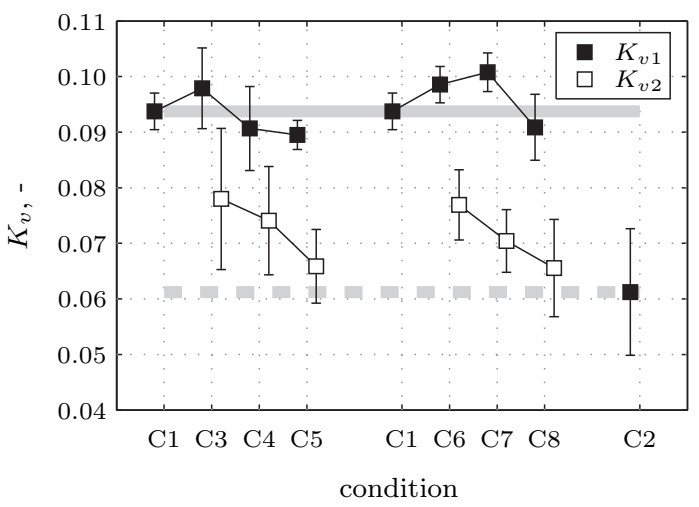

(c) Tim of maximum growth

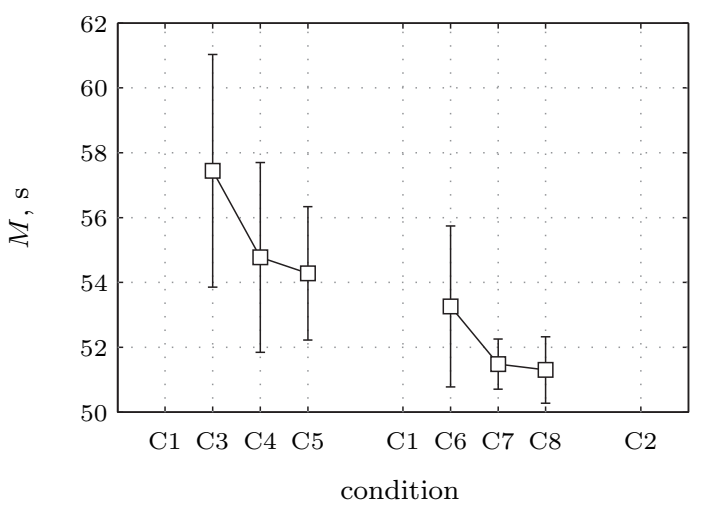

(b) Lead time constant

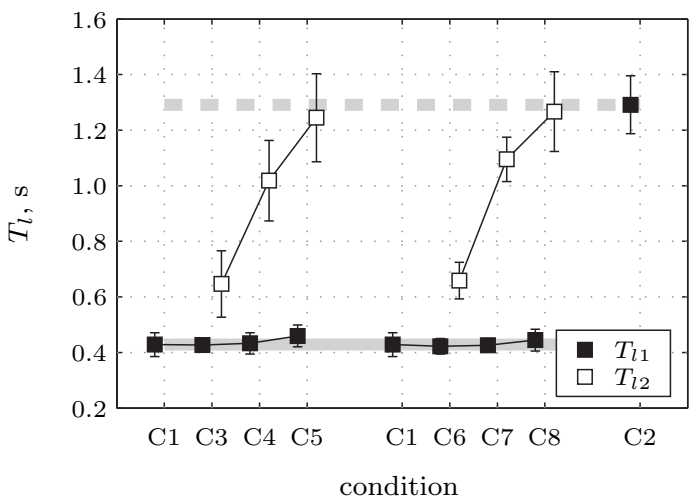

(d) Growth rate

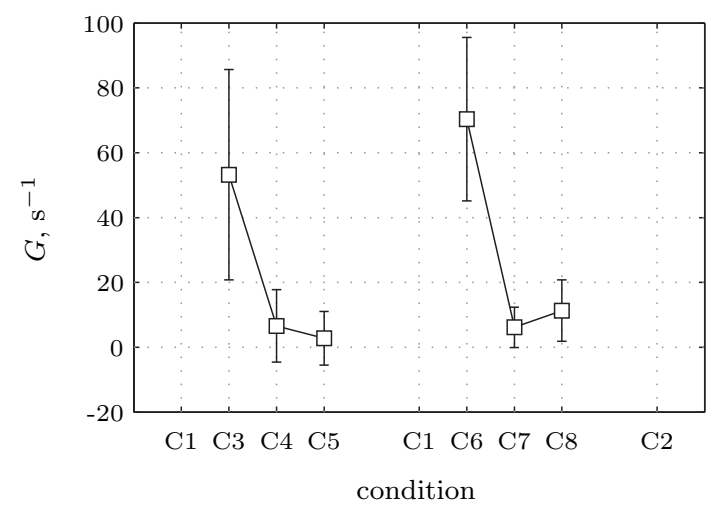

(e) Visual delay

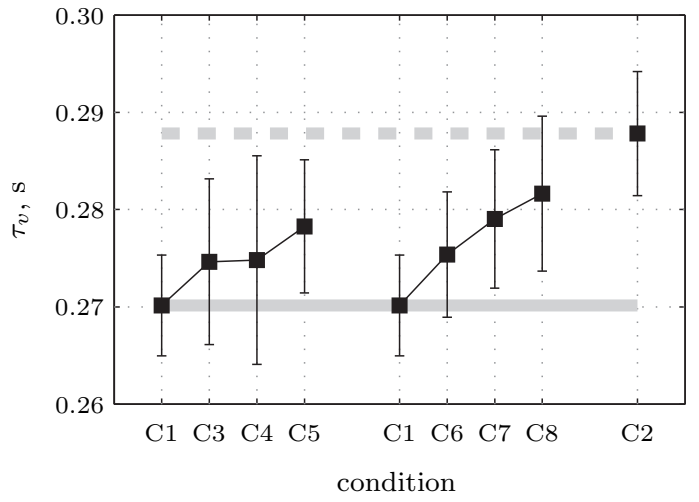

(f) Neuromuscular damping

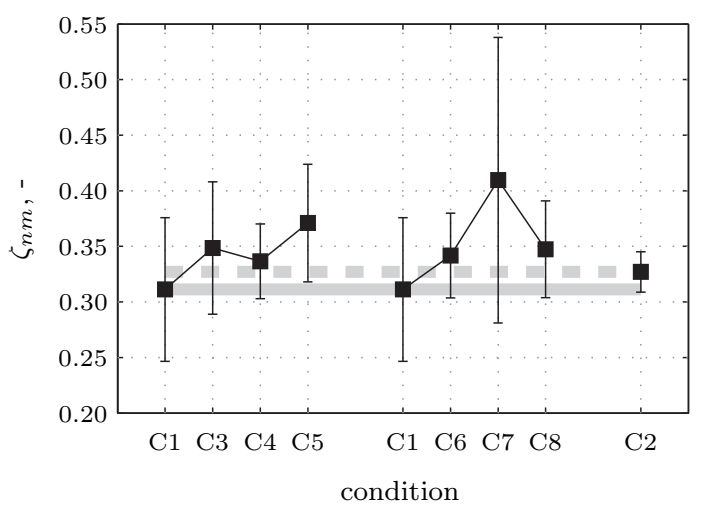

(g) Neuromuscular frequency

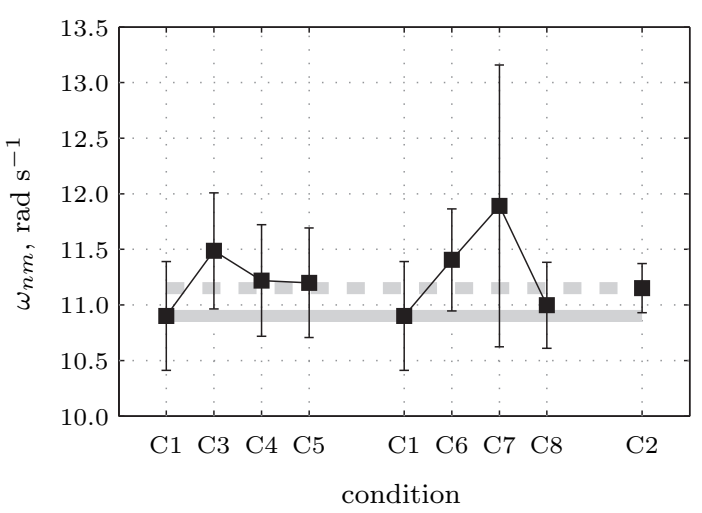

Figure 12. Means and $95 \%$ confidence intervals of the pitch pilot model parameters. 
conditions with a variation in pitch dynamics, however, not for the conditions with only a variation in roll dynamics. This indicates that control behavior in the pitch axis is influenced by the time-varying aircraft dynamics in roll.

Comparing the visual gains between the roll and pitch axes (Fig. 11a with Fig. 12a) it was found that the initial visual gain $K_{v 1}$ was significantly higher in the pitch axis $[F(1,8)=7.469, p<0.05]$. The final visual gain $K_{v 2}$ is not significantly different in the roll or pitch axis $[F(1,8)=0.514, p>0.05]$.

From Fig. 11b it can be observed that in the roll axis the visual lead $T_{l 1}$ is significantly higher in $\mathrm{C} 2$ compared to $\mathrm{C} 1[F(1,8)=48.471, p<0.001]$. Based on the characteristics of the aircraft dynamics this was an expected result and also supports findings in previous studies. The initial visual lead $T_{l 1}$ was not affected by a change in dynamics or the growth rate, again an expected result as this parameter describes control behavior for the stable dynamics $H_{c 1}$ in all conditions, except $\mathrm{C} 2$. The change in dynamics introduced a significant effect in the final lead time constant $T_{l 2}[F(2,16)=38.755, p<0.001]$. For the conditions with a change in roll dynamics, $T_{l 2}$ is higher and resembles $T_{l 1}$ in condition C2. A comparison of $T_{l 1}$ with $T_{l 2}$ showed a significantly higher value is being found for $T_{l 2}[F(1,8)=345.814, p<0.001]$.

The effects for the visual lead time constant in the pitch axis were similar to the effects in the roll axis (Fig. 12b). In the pitch axis, the lead time constant $T_{l 1}$ is also significantly higher for condition $\mathrm{C} 2$ compared to $\mathrm{C} 1[F(1,8)=190.593, p<0.001]$. The change in dynamics in roll or pitch, or the growth rate did not introduce a significant effect in $T_{l 1}$. The change in dynamics did introduce a significant effect in the final lead time constant $T_{l 2}[F(2,16)=50.122, p<0.001]$. The lead time constant for the conditions with a change in pitch dynamics approaches the value of the lead time constant found for C2. Comparing $T_{l 1}$ with $T_{l 2}$ in the pitch axis reveals that $T_{l 2}$ is significantly higher $[F(1,8)=302.983, p<0.001]$. In the conditions with only a change in roll dynamics (C3 and $\mathrm{C} 6)$, the lead time constant in the pitch axis is significantly higher, indicating a cross coupling effect.

When the lead time constant is compared between the roll and pitch axes (Fig. 11b with Fig. 12b), it is found that the initial lead time constant $T_{l 1}$ is not significantly different between the two axes $[F(1,8)=$ $0.884, p>0.05]$. The final lead time constant $T_{l 2}$ is significantly higher in the pitch axis compared to the roll axis $[F(1,8)=8.720, p<0.05]$.

Figs. $11 \mathrm{c}$ and $12 \mathrm{c}$ depict the time of maximum growth for the roll and pitch axis, respectively. No significant effects were found for the change in roll or pitch dynamics. In both axes the growth rate introduced a significant effect, $[F(1,8)=16.573, p<0.01]$ and $[F(1,8)=15.529, p<0.01]$. For the higher growth rate, representing the step-like change, the time of maximum growth is smaller, indicating that the change in pilot control behavior occurs sooner after the change in aircraft dynamics at $t=50 \mathrm{~s}$. No significant differences in $M$ were found between the roll and pitch axes $[F(1,8)=0.099, p>0.05]$.

The growth rate $G$ of the pilot visual gain and lead time constant is presented in Fig. 11d for the roll axis and Fig. 12d for the pitch axis. The value of the growth rate had an upper bound of 100 in the estimation procedure. In both the roll and pitch axes, the growth rate of the aircraft dynamics did not affect the growth rate of the change in pilot dynamics, $[F(1,8)=1.078, p>0.05]$ and $[F(1,8)=1.344, p>0.05]$. The change in dynamics did not introduce a significant effect in the roll axis $[F(2,16)=3.063, p>0.05]$, however in the pitch axis the growth rate is significantly higher in the conditions with only a change in roll dynamics $[F(2,16)=60.643, p<0.001]$. Comparing the growth rates between both axes (Fig. 11d with Fig. 12d), the growth rate in the pitch axis is significantly lower $[F(1,8)=36.409, p<0.001]$. Note that the error bars for the growth rate are relatively large, indicating large variations between subjects. This is most likely an effect from the lower accuracy of this parameter in the estimation procedure, as found in Sec. III.B.

The pilot visual time delay $\tau_{v}$ is depicted in Figs. 11e and 12e for the roll and pitch axes, respectively. In the roll axis, the visual time delay is not significantly different between the two conditions with constant dynamics $(\mathrm{C} 1$ and $\mathrm{C} 2)[F(1,8)=0.259, p>0.05]$. This supports the assumption that this parameter does not change over time when the aircraft dynamics change as described in Sec. II. In the pitch axis, the visual time delay is significantly different between conditions $\mathrm{C} 1$ and $\mathrm{C} 2[F(1,8)=18.950, p<0.01]$. However, note that the difference is only $20 \mathrm{~ms}$. In the roll axis, the visual time delay is significantly affected by a change in aircraft pitch dynamics $[F(1,8)=23.923, p<0.01]$. When a change in pitch dynamics occurs, the time delay in roll is significantly lower. The change in roll dynamics or the growth rate did not change the time delay significantly, $[F(1,8)=2.685, p>0.05]$ and $[F(1,8)=0.062, p>0.05]$. Comparing the time delay between the roll and pitch axes (Fig. 11e with Fig. 12e), the time delay in the pitch axis is significantly higher $[F(1,8)=19.753, p<0.01]$.

Figs. 11f and $12 \mathrm{f}$ depict the neuromuscular damping for the roll and pitch axis, respectively. In both 
axis, the damping is not different between conditions $\mathrm{C} 1$ and $\mathrm{C} 2$, supporting the assumption to define this parameter as a constant parameter, $[F(1,8)=1.851, p>0.05]$ and $[F(1,8)=0.229, p>0.05]$. In the roll axis, no significant effects were found for the change in roll or pitch dynamics, or the growth rate $([F(1,8)=$ $0.135, p>0.05],[F(1,8)=4.945, p>0.05]$ and $[F(1,8)=0.757, p>0.05])$. The change in dynamics and the growth rate also did not introduce a significant effect in the pitch axis $([F(1,8)=0.563, p>0.05],[F(1$, $8)=1.039, p>0.05]$ and $[F(1,8)=1.060, p>0.05])$. Comparing the neuromuscular damping between the two axes, the damping was found to be significantly lower in the pitch axis, $[F(1,8)=16.467, p<0.01]$.

The neuromuscular frequency is given in Figs. $11 \mathrm{~g}$ and $12 \mathrm{~g}$ for the roll and pitch axes, respectively. There was no significant difference between conditions $\mathrm{C} 1$ and $\mathrm{C} 2$, supporting the assumption of a constant parameter, $[F(1,8)=0.706, p>0.05]$ and $[F(1,8)=0.976, p>0.05]$. In the roll axis, the neuromuscular frequency was significantly higher when there was a change in roll dynamics present $[F(1,8)=9.277, p<$ $0.05]$ and significantly lower when there was a change in pitch dynamics present $[F(1,8)=55.881, p<$ $0.001]$. The growth rate did not introduce a significant effect, $[F(1,8)=1.313, p>0.05]$. In the pitch axis, no significant effects were found for the change in roll or pitch dynamics, or the growth rate $([F(1,8)=$ $0.053, p>0.05],[F(1,8)=0.226, p>0.05]$ and $[F(1,8)=0.641, p>0.05])$. Comparing the neuromuscular frequency between the two axes, the frequency was found to be significantly higher in the pitch axis, $[F(1$, 8) $=44.461, p<0.001]$.

An example of the time-varying pilot visual gain and visual lead time constant as defined by the parameters estimated using MLE is depicted in Fig. 13 for both the roll and pitch axes of condition C8 for one pilot. The significant trends identified in Figs. 11 and 12 are clearly visible in the parameter functions.

(a) Visual gain

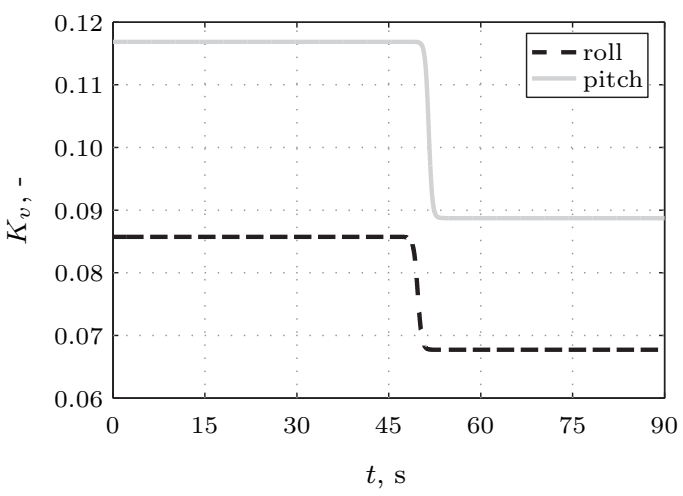

(b) Visual lead time constant

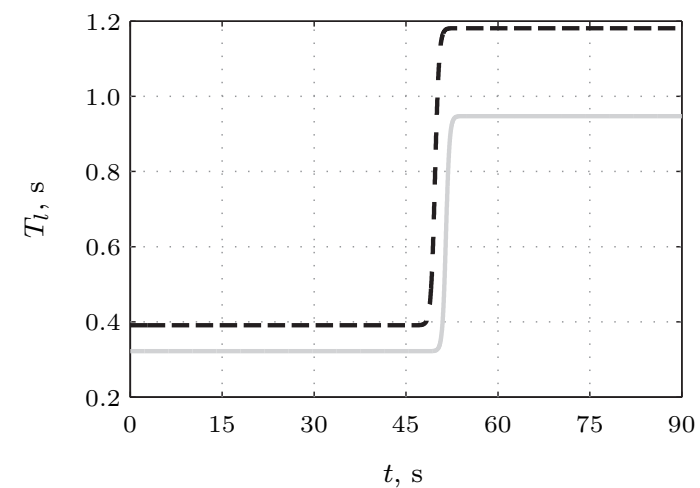

Figure 13. Time-varying pilot model parameters (1 pilot, condition C8).

\section{Pilot-Vehicle Open Loop}

The crossover frequency and phase margin of the pilot-vehicle open loop provide insight into the performance and stability margins of the pilot-vehicle system. The crossover frequency $\omega_{c}$, is the frequency where the magnitude of the open-loop response has a magnitude of 1 . The corresponding phase margin $\varphi$ is the phase difference with $-180 \mathrm{deg}$ at the crossover frequency. Fig. 14 provides an example of the open-loop frequency response for the roll axis of condition $\mathrm{C} 8$ for one pilot. A clear increase in open-loop gain and decrease in effective time-delay $\tau_{e}$ can be seen after the change in aircraft dynamics. The result is an increase in crossover frequency and a decrease in phase margin, as depicted in Fig. 15. This figure also provides the time-varying crossover frequency and phase margin for the pitch axis.

\section{Discussion}

A parameter estimation technique based on MLE was used to identify a pilot model with time-dependent parameter functions characterizing time-varying pilot control behavior. The technique was used to estimate the pilot model parameters using experimental data from a multi-axis disturbance-rejection task.

Several assumptions were made with respect to the time-varying pilot model. The first was the type of parameter function to describe the time-varying parameters, which was assumed to be equivalent to the parameter function used to vary the aircraft dynamics over time. Given the aircraft dynamics used in the 
(a) Open-loop magnitude

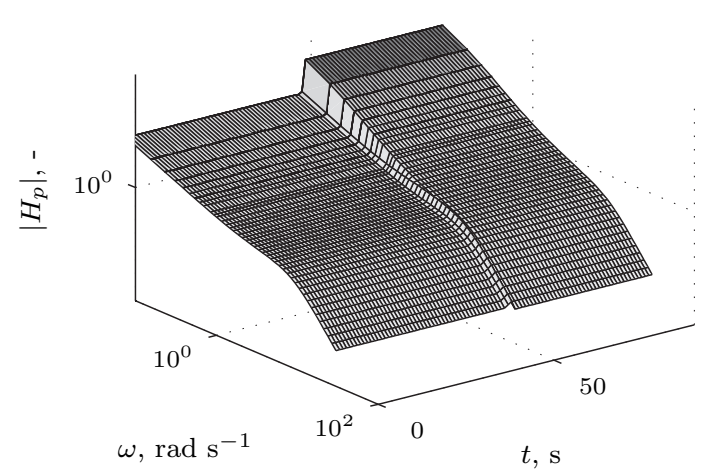

(b) Open-loop phase

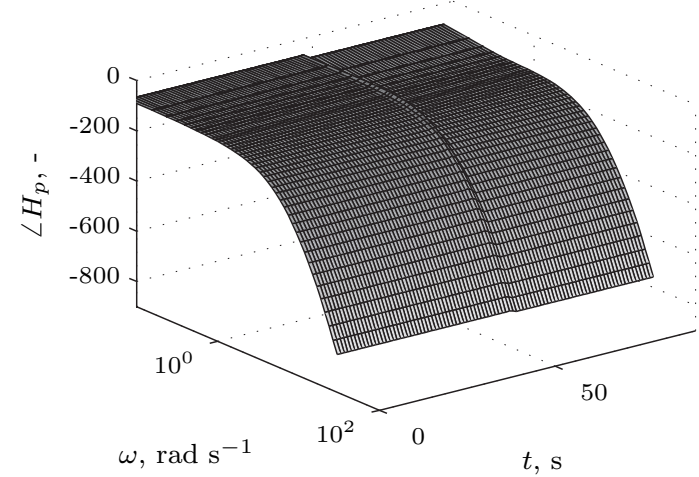

Figure 14. Time-varying open-loop frequency response for the roll axis (1 pilot, condition C8).

(a) Crossover frequency

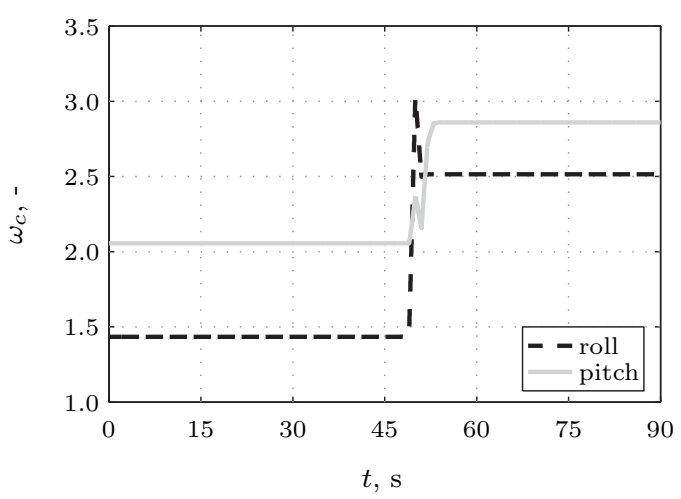

(b) Phase margin

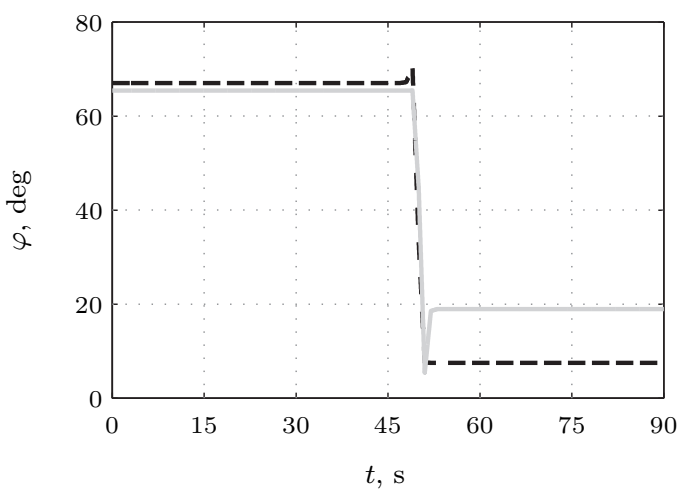

Figure 15. Crossover frequency and phase margin as a function of time (1 pilot, condition C8).

experiment, this was a straightforward choice. However, if time-varying pilot control behavior needs to be identified when little is known about the characteristics of the time-varying controlled dynamics, the selection of the most appropriate parameter function can be more difficult. It requires incorporating the available knowledge of the time-varying aspect of the controlled dynamics and careful inspection of the measured data.

The second assumption is the introduction of an equivalent time of maximum growth and growth rate for both parameter functions in the pilot model. This means that the time-varying parameters of the model change at the same time and with the same rate with only their initial and final values differing. This reduces the number of parameters to be estimated, resulting in more accurate parameter estimates. However, this assumption may not hold for all applications and must be considered in conjunction with the selection of the parameter function type.

The final assumption is to only let the pilot equalization vary over time, in this case the visual gain and visual lead time constant. The remaining parameters - the visual time delay, the neuromuscular damping, and the neuromuscular frequency - were assumed to be constant over time. This assumption was also necessary to reduce the number of parameters to be estimated in the MLE procedure, increasing the accuracy. Previous research has shown that pilots mainly adapt their equalization dynamics when controlling the different controlled dynamics used in the current study. ${ }^{15}$ The assumption was validated by comparing constant pilot control behavior between two reference conditions with constant aircraft dynamics, C1 and C2. The aircraft dynamics in these conditions are the same as the initial and final aircraft dynamics in conditions C3-C8. Comparing the estimated pilot model parameters between $\mathrm{C} 1$ and $\mathrm{C} 2$ shows that only the visual gain and lead time constant were significantly different between the two conditions.

To validate the accuracy of the pilot model, the VAF was calculated for the roll and pitch control signal. The VAF was generally around $70 \%$ for the roll axis and around $82 \%$ for the pitch axis. These values are slightly lower compared to values found in single-axis experiments. This was an expected result, since in multi-axis control tasks the pilot's attention needs to be divided between the two axes, resulting in higher 
levels of pilot remnant. The higher VAF for the pitch axis indicates that pilot control behavior was more linear in the pitch axis (that is, remnant levels were lower) compared to the roll axis. This could be due to the fact that a pitch disturbance is more visible than a roll disturbance due to the nature of the primary flight display used in the experiment (a roll disturbance is depicted by an angle deviation, while a pitch disturbance is depicted by a vertical displacement), or simply by the fact that pilots paid more attention to pitch compared to roll.

Furthermore, as hypothesized in Sec. IV.B, the pilot model VAF for the unstable double-integrator-like dynamics $H_{c 2}$ was higher compared to the pilot model VAF for the single-integrator-like dynamics $H_{c 1}$. This was also found in previous experiments. ${ }^{17}$ Finally, for the conditions with time-varying aircraft dynamics (C3-C8), a constant pilot model with 5 parameters $H_{p}(s)$ was identified in addition to the time-varying pilot model with 9 parameters $H_{p}(s, t)$, to verify if the added time-varying parameter functions provide a higher overall model accuracy. This was indeed the case, for all conditions with time-varying aircraft dynamics, the time-varying pilot model had a significant higher VAF compared to the constant model. The difference in VAF was greater in the pitch axis (approximately $7 \%$ ) compared to the roll axis (approximately $3 \%$ ).

The accuracy of the MLE procedure and the selected pilot model structure was also analyzed in a Monte Carlo analysis before the start of the experiment. This analysis showed that the final values of the parameter functions and the growth rate have the highest bias and variance, and that bias and variance increase when pilot remnant intensity increases. The lower accuracy of the growth factor can be explained by the fact that this value is only affected by data from a small time window. In addition, the growth rate does not have an upper limit; that is, for values of approximately 10 to infinity, the parameter function is a step function. For this reason, the growth rate had an upper bound of 100 in the estimation procedure.

The independent variables - the changing aircraft dynamics in the roll and pitch axes, and the growth rate of the dynamics - introduced significant adaptations in pilot control behavior. Mainly the parameters defining the time-dependent sigmoid functions were significantly affected. The main effect was in the axis of the changing aircraft dynamics. However, significant effects were also found in the axis other than the axis of the changing aircraft dynamics. This indicates some cross coupling in pilot control behavior between the two axes, and might warrant the addition of a cross coupling term in a pilot model predicting multi-axis control behavior, even when the controlled dynamics in the two axis are not coupled.

When comparing the results across conditions between the roll and pitch axes, it was found that performance is higher and control activity is lower in the pitch axis. In addition, the average initial visual gain, final visual lead time constant, time delay, and neuromuscular frequency were all significantly higher for the pitch axis, while the neuromuscular damping was significantly lower. Note that the aircraft dynamics and forcing functions are equivalent in both controlled axes, indicating that these effects were caused by differences in display and joystick characteristics between the two axis, or differences in human perception and control processes between the two axes.

Most studies on pilot control behavior use control tasks in a single axis and assume constant pilot control behavior. These assumptions reduce the applicability of the results from these studies to real piloting tasks, as these are often performed in multiple axes and are not constant over time. In this paper time-varying pilot control behavior was accurately identified using data from a multi-axis control task, thus expanding the scope of pilot control behavior research. Further research is required to investigate the use of different types of parameter functions to allow for more complex parameter variations in time. In addition, time-variations in parameters other than the pilot equalization, such as time-varying perceptual time delays, should be investigated.

\section{Conclusions}

A maximum likelihood parameter estimation method was used to identify a pilot model with timedependent sigmoid functions to characterize time-varying human control behavior. An experiment was performed with 9 general aviation pilots performing a simultaneous roll and pitch control task with timevarying aircraft dynamics. In 8 different conditions the controlled axis containing the time-varying dynamics and the growth factor of the change in dynamics was varied. The MLE method used was able to estimate most parameters of the time-varying pilot model from the experiment data accurately. However, the growth factor of the sigmoid functions had the lowest estimation accuracy, as it is unbounded for higher values due to the nature of the sigmoid function. Time-varying pilot control behavior in both the roll and pitch axes was significantly affected by the axis containing the time-varying aircraft dynamics and the growth factor. The 
main effect was found in the axis that contained the time-varying aircraft dynamics. However, significant effects were also found in the opposite axis. This indicates that cross coupling exists in pilot control behavior between the two axes. Although the same aircraft dynamics and forcing functions are used in both axes of control, pilot performance was higher and control activity was lower in pitch compared to roll. This was also reflected by significantly different pilot control behavior in both axes.

\section{References}

${ }^{1}$ Hess, R. A., "Modeling Pilot Control Behavior with Sudden Changes in Vehicle Dynamics," Journal of Aircraft, Vol. 46, No. 5, Sept.-Oct. 2009, pp. 1584-1592.

${ }^{2}$ Fletcher, J. W., Lusardi, J., Mansur, M., Moralis, E., and Robinson, D., "UH-60M Upgrade Fly-By- Wire Flight Control Risk Reduction Using the RASCAL JUH-60A In-Flight Simulator," Proceedings of the AHS 64th Annual Forum, Montreal, Canada, April29 2008.

${ }^{3}$ Young, L. R., "On Adaptive Manual Control," Ergonomics, Vol. 12, No. 4, 1969, pp. 635-675.

${ }^{4}$ McRuer, D. T., Graham, D., Krendel, E. S., and Reisener, W., "Human Pilot Dynamics in Compensatory Systems. Theory, Models and Experiments With Controlled Element and Forcing Function Variations," Tech. Rep. AFFDL-TR-65-15, Wright Patterson AFB (OH): Air Force Flight Dynamics Laboratory, 1965.

${ }^{5}$ Stapleford, R. L., Peters, R. A., and Alex, F. R., "Experiments and a Model for Pilot Dynamics with Visual and Motion Inputs," NASA Contractor Report NASA CR-1325, NASA, 1969.

${ }^{6}$ Nieuwenhuizen, F. M., Zaal, P. M. T., Mulder, M., van Paassen, M. M., and Mulder, J. A., "Modeling Human Multichannel Perception and Control Using Linear Time-Invariant Models," Journal of Guidance, Control, and Dynamics, Vol. 31, No. 4, July-Aug. 2008, pp. 999-1013.

${ }^{7}$ Zaal, P. M. T., Pool, D. M., Chu, Q. P., van Paassen, M. M., Mulder, M., and Mulder, J. A., "Modeling Human Multimodal Perception and Control Using Genetic Maximum Likelihood Estimation," Journal of Guidance, Control, and Dynamics, Vol. 32, No. 4, July-Aug. 2009, pp. 1089-1099.

${ }^{8}$ Thompson, P. M., Klyde, D. H., and Brenner, M. J., "Wavelet-Based Time-Varying Human Operator Models," Proceedings of the AIAA Atmospheric Flight Mechanics Conference and Exhibit, Montreal (CA), No. AIAA-2001-4009, 6-9 Aug. 2001.

${ }^{9}$ Thompson, P. M. and Klyde, D. H., "Exploration of the Properties of Analytic Wavelets for Systems Analysis," Proceedings of the AIAA Atmospheric Flight Mechanics Conference and Exhibit, Monterey (CA), No. AIAA-2002-4707, Aug. 5-8 2002.

${ }^{10}$ Thompson, P. M., Klyde, D. H., Bachelder, E. N., Rosenthal, T. J., and Brenner, M. J., "Development of Wavelet-Based Techniques for Detecting Loss of Control," Proceedings of the AIAA Atmospheric Flight Mechanics Conference and Exhibit, Providence (RI), No. AIAA-2004-5064, Aug. 16-19 2004.

${ }^{11}$ Zaal, P. M. T. and Sweet, B. T., "Estimation of Time-Varying Pilot Model Parameters," Proceedings of the AIAA Modeling and Simulation Technologies Conference and Exhibit, Portland (OR), No. AIAA-2011-6474, 8-11 Aug. 2011.

${ }^{12}$ Stapleford, R. L., McRuer, D. T., and Magdaleno, R., "Pilot Describing Function Measurements in a Multiloop Task," IEEE Transactions on Human Factors in Electronics, Vol. HFE-8, No. 2, 1967, pp. 113-125.

${ }^{13}$ van Lunteren, A., Identification of Human Operator Describing Function Models with One or Two Inputs in Closed Loop Systems, Doctoral dissertation, Faculty of Aerospace Engineering, Delft University of Technology, 1979.

${ }^{14}$ van der Vaart, J. C., Modelling of Perception and Action in Compensatory Manual Control Tasks, Doctoral dissertation, Faculty of Aerospace Engineering, Delft University of Technology, 1992.

${ }^{15}$ Breur, S. W., Pool, D. M., van Paassen, M. M., and Mulder, M., "Effects of Displayed Error Scaling in Compensatory Roll-Axis Tracking Tasks," Proceedings of the AIAA Guidance, Navigation, and Control Conference and Exhibit, Toronto (ON), Canada, No. AIAA-2010-8091, 2-5 Aug. 2010.

${ }^{16}$ Groot, T., Damveld, H. J., Mulder, M., and van Paassen, M. M., "Effects of Aeroelasticity on the Pilots Psychomotor Behavior," Proceedings of the AIAA Atmospheric Flight Mechanics Conference and Exhibit, Keystone (CO), No. AIAA-20066494, 21-24 Aug. 2006.

${ }^{17}$ Pool, D. M., Zaal, P. M. T., Damveld, H. J., van Paassen, M. M., van der Vaart, J. C., and Mulder, M., "Modeling WideFrequency-Range Pilot Equalization for Control of Aircraft Pitch Dynamics," Journal of Guidance, Control, and Dynamics, Vol. 34, No. 5, Sept.-Oct. 2011, pp. 1529-1542.

${ }^{18}$ Loftus, G. R. and Masson, M. E. J., "Using Confidence Intervals in Within-Subject Designs," Psychonomic Bulletin 83 Review, Vol. 1, No. 4, 1994, pp. 476-490. 